\title{
Bassin d'oued Serrat : terrils et rejets domestiques, reconnaissance des métaux lourds et polluants, impact sur les eaux souterraines (nord-ouest de la Tunisie) The Oued Serrat: Mining activity, ground water and dispersion of base metals in natural environments (North-western
} Tunisia)

\author{
Ammar Mlayah, Eduardo Anselmo Ferreira Da Silva, Nouri Hatira, Salah \\ Jellali, Fethi Lachaal, Abdelkrim Charef, Fernando Noronha et Chedly Ben \\ Hamza
}

Volume 24, numéro 2, 2011

Reçu le 10 février 2010, accepté le 7 septembre 2010

URI : https://id.erudit.org/iderudit/1006109ar

DOI : https://doi.org/10.7202/1006109ar

Aller au sommaire du numéro

Éditeur(s)

Université du Québec - INRS-Eau, Terre et Environnement (INRS-ETE)

ISSN

0992-7158 (imprimé)

1718-8598 (numérique)

Découvrir la revue

Citer cet article

Mlayah, A., Ferreira Da Silva, E. A., Hatira, N., Jellali, S., Lachaal, F., Charef, A., Noronha, F. \& Ben Hamza, C. (2011). Bassin d'oued Serrat : terrils et rejets domestiques, reconnaissance des métaux lourds et polluants, impact sur les eaux souterraines (nord-ouest de la Tunisie). Revue des sciences de l'eau / Journal of Water Science, 24(2), 159-175. https://doi.org/10.7202/1006109ar

\section{Résumé de l'article}

Le présent travail visait à déterminer l'impact de rejets de deux mines de fer et de phosphates sur la qualité physicochimique de l'eau d'un système aquifère alluvial (nord-ouest de la Tunisie). Des échantillons de terrils, de sols ainsi que de l'eau de puits captant la nappe ont été prélevés et analysés. Les résultats d'analyses des échantillons de terrils ont montré que cette matrice renferme des concentrations remarquables en métaux lourds (essentiellement le $\mathrm{Cd}, \mathrm{Cr}$, $\mathrm{Pb}$ et $\mathrm{Zn}$ ). Ces éléments métalliques ont été également détectés à des concentrations non négligeables dans la fraction fine des sols situés en aval des rejets miniers, suite à son transport éolien et hydrique. En ce qui concerne les eaux de la nappe, il s'avère que le transport des métaux issus des terrils est totalement atténué par les éléments constituant le sous-sol. Ces eaux, de bonne qualité géochimique, sont toutefois assez contaminées en nitrates dû aux pratiques agricoles intenses et à l'absence de systèmes d'assainissement urbains. 


\title{
BASSIN D'OUED SERRAT : TERRILS ET REJETS DOMESTIQUES, RECONNAISSANCE DES MÉTAUX LOURDS ET POLLUANTS, IMPACT SUR LES EAUX SOUTERRAINES (NORD-OUEST DE LA TUNISIE)
}

The Oued Serrat: Mining activity, ground water and dispersion of base metals in natural environments (North-western Tunisia)

\author{
Ammar MLAYAH ${ }^{\prime *}$, Eduardo Anselmo FERREIRA DA SILVA², Nouri HATIR $A^{3}$, Sal aH JELLALI', \\ FETHI LACHAAL', ABDELKRIM CHAREF', FERNANDO NORONHA ${ }^{4}$ ET CHEDLY BEN HAMZ $A^{5}$ \\ ${ }^{1}$ Laboratoire Géoressources CERTE, Route Touristique de Soliman, 8020, Soliman, Tunisie \\ ${ }^{2}$ GeoBioTec-Geobiosciences, Geotechnologies and Geoengineering Research Center, \\ Universidade de Aveiro, 3810-193 Aveiro, Portugal \\ ${ }^{3}$ Faculté des Sciences de Gabes, Cité Erriadh, 6072, Gabes \\ ${ }^{4}$ Centro de Geologia, Rua Campo Alegre, 4769-007, Porto, Portugal \\ ${ }^{5}$ Faculté des Sciences de Bizerte, 7021, Jarzouna, Tunisie \\ Reçu le 10 février 2010, accepté le 7 septembre 2010
}

\section{RÉSUMÉ}

Le présent travail visait à déterminer l'impact de rejets de deux mines de fer et de phosphates sur la qualité physicochimique de l'eau d'un système aquifere alluvial (nord-ouest de la Tunisie). Des échantillons de terrils, de sols ainsi que de l'eau de puits captant la nappe ont été prélevés et analysés. Les résultats d'analyses des échantillons de terrils ont montré que cette matrice renferme des concentrations remarquables en métaux lourds (essentiellement le $\mathrm{Cd}, \mathrm{Cr}, \mathrm{Pb}$ et $\mathrm{Zn}$ ). Ces éléments métalliques ont été également détectés à des concentrations non négligeables dans la fraction fine des sols situés en aval des rejets miniers, suite à son transport éolien et hydrique. En ce qui concerne les eaux de la nappe, il s'avère que le transport des métaux issus des terrils est totalement atténué par les éléments constituant le sous-sol. Ces eaux, de bonne qualité géochimique, sont toutefois assez contaminées en nitrates dû aux pratiques agricoles intenses et à l'absence de systèmes d'assainissement urbains.

\section{Mots clés : terrils miniers, métaux lourds, nappe} phréatique, pollution, géochimie.

\begin{abstract}
The aims of this work were to determine the impacts of releases from two iron and phosphate mines (North West Tunisia) on the physico-chemical water composition of the related alluvial aquifer system. Samples from tailings, soil and water wells were collected and analyzed. The analytical results of tailing samples showed that this matrix contains high concentrations of heavy metals (mainly $\mathrm{Cd}, \mathrm{Cr}, \mathrm{Pb}$ and $\mathrm{Zn}$ ). These metals were also detected at significant levels in the fine soil fraction downstream from the mine waste discharge due to transport by wind and water. The heavy metal concentrations in groundwater showed that the metal transport from the tailings is completely attenuated by the soil constituents. Overall, the physic-chemical quality of the groundwater is good, except for nitrates, which are present at relatively high concentrations due to intensive agricultural practices and the absence of urban wastewater treatment plants.
\end{abstract}

Key words: mine tailings, heavy metals, groundwater pollution, geochemistry.

*Auteur pour correspondance:

Téléphone: $\quad+21679325750$

T.élécopieur: +216 79325802

Courriel: ammarmlayab@yahoo.fr 


\section{INTRODUCTION}

En Tunisie, la richesse en eau est inégalement répartie à travers le pays, $60 \%$ est localisée au Nord, $18 \%$ dans le Centre et $22 \%$ au Sud (DGRE, 2005). Le Nord du pays est également d'un grand intérêt minier; il renferme un grand nombre de gîtes et indices miniers (plus de $95 \%$ ), représentant l'essentiel du potentiel $\mathrm{Pb}-\mathrm{Zn}-\mathrm{Fe}-\mathrm{Ba}$ (HATIRA et al., 1990).

La pollution des eaux par les métaux, dans les zones minières, a été principalement associée à l'oxydation des sulfures minéraux exposés aux conditions atmosphériques. Cette oxydation engendre à la fois une acidification du milieu et un enrichissement en métaux dissous (SALOMONS, 1995). Les eaux émanées des mines abandonnées, des galeries souterraines et des terrils peuvent s'infiltrer vers les eaux souterraines à travers les couches sédimentaires, les fissures et les micropores et engendrent une pollution du milieu aquifère récepteur (FURRER et al., 2002). Cette pollution a été bien enregistrée dans plusieurs pays aux confins de sites miniers (CAPPUYNS et al., 2006; LEE et CHON, 2006; PRATAS et al., 2005).

Le transfert des métaux dans le sous-sol dépend non seulement de leurs caractéristiques physico-chimiques, mais également des propriétés du milieu souterrain (SIPOS et al., 2005). En effet, le sol est une matrice complexe, dans lequel l'activité de l'ion métallique dépend de sa spéciation chimique, des caractéristiques du sol telles que la capacité d'échange cationique, la teneur en matière organique, les teneurs en oxydes et le $\mathrm{pH}$. Ces paramètres ont été démontrés être déterminants dans les processus chimiques de précipitation, de sorption et de complexation (COVELO et al., 2004; SIPOS et al., 2005).

Par ailleurs, le développement de l'agriculture a été pénalisé par une dégradation de la qualité de l'eau suite à l'utilisation intense des engrais chimiques et des fertilisants. Ainsi, la dégradation de la qualité des eaux de surface et souterraines par les éléments azotés, phosphorés et métalliques, induite par l'activité agricole, a été largement vérifiée dans le monde (COSTA et al., 2002; HADAS et al., 1999; PATNI et al., 1998; TRABELSI et al., 2007). L'élevage participe, lui aussi, d'une manière remarquable à la pollution des eaux (KRAPAC et al., 2002).

Le présent travail a pour objectifs de déterminer : 1) l'impact du lessivage des métaux lourds de deux rejets des mines de fer et de phosphates sur les sols environnants et les eaux de la nappe souterraine; et 2) l'effet des activités agricoles et domestiques sur la qualité géochimique de la nappe.

\section{PRÉSENTATION DE LA ZONE D’ÉTUDE}

La plaine de Kalaa Khasba (K. Kh) se situe au nordouest de la Tunisie, à une distance de $220 \mathrm{~km}$ de Tunis, capitale du pays, à une altitude qui varie entre $700 \mathrm{~m}$ au Sud et $500 \mathrm{~m}$ dans sa partie septentrionale. Elle a une superficie de l'ordre de $230 \mathrm{~km}^{2}$ et est limitée au Nord par la terminaison du Jbel El Hout, au Sud par le plateau de Tala qui culmine à $1156 \mathrm{~m}$ et les faibles reliefs de K. Kh. Ses bordures est et sud-est sont bien marquées par le jbel Jerissa, Boulahnech (1 229 m) et le Jbel Zaba (1 271 m). À l'Ouest, le relief est plus atténué jusqu'au plateau de Kalaat Senan (1 $271 \mathrm{~m}$ ). La plaine de K. Kh. est décrite par différents auteurs (JAUZIN, 1967; ZEBIDI, 1976) comme étant une plaine d'effondrement développée en travers de la direction majeure de l'Atlas tunisien (Sud-Ouest, Nord-Est), (Figure 1).

Le climat qui règne dans la région est semi-aride, caractérisé par une saison humide qui s'étend du mois de septembre au mois d'avril, avec des précipitations intenses en automne. La moyenne pluviométrique annuelle, durant les vingt dernières années, est de $360 \mathrm{~mm}$ à K. Kh. et $400 \mathrm{~mm}$ à Tala. En général, $50 \%$ des précipitations annuelles sont enregistrées pendant la période hivernale. La saison sèche s'étend du mois de mai à octobre, avec des précipitations mensuelles relativement faibles. La température annuelle moyenne varie entre $15,2^{\circ} \mathrm{C}$ et $19,4{ }^{\circ} \mathrm{C}$, avec un maximum durant les mois de juillet et d'août (de $31,3{ }^{\circ} \mathrm{C}$ et $36,7^{\circ} \mathrm{C}$ respectivement). La température moyenne minimale est enregistrée au mois de janvier avec des chutes de neige, en particulier sur les hauts plateaux de Tala.

Cette plaine alluviale destinée dans sa totalité à l'agriculture (céréale, culture maraîchère, arboriculture) et à l'élevage, est localisée entre deux rejets miniers : au SudOuest, celui de la mine abandonnée de phosphate de K. Kh. et au Nord, celui de la mine de fer de Jérissa, actuellement en production. L'oued Sarrath forme le principal cours d'eau de la plaine (Figure 1). Cet oued semble jouer un double rôle hydrodynamique dans la plaine; il l'alimente en amont par ses différents affluents, pour être son exutoire en aval (ZEBIDI, 1976). Par ailleurs, cet oued draine les différents rejets miniers et domestiques des agglomérations de Tala, Jérissa, Hidra, $\mathrm{K}$. Kh. et le reste des hameaux implantés dans son bassin versant. La zone d'étude renferme une nappe phréatique dont le niveau statique ne dépasse pas, par endroits, les dix mètres, ce qui la rend particulièrement vulnérable. 


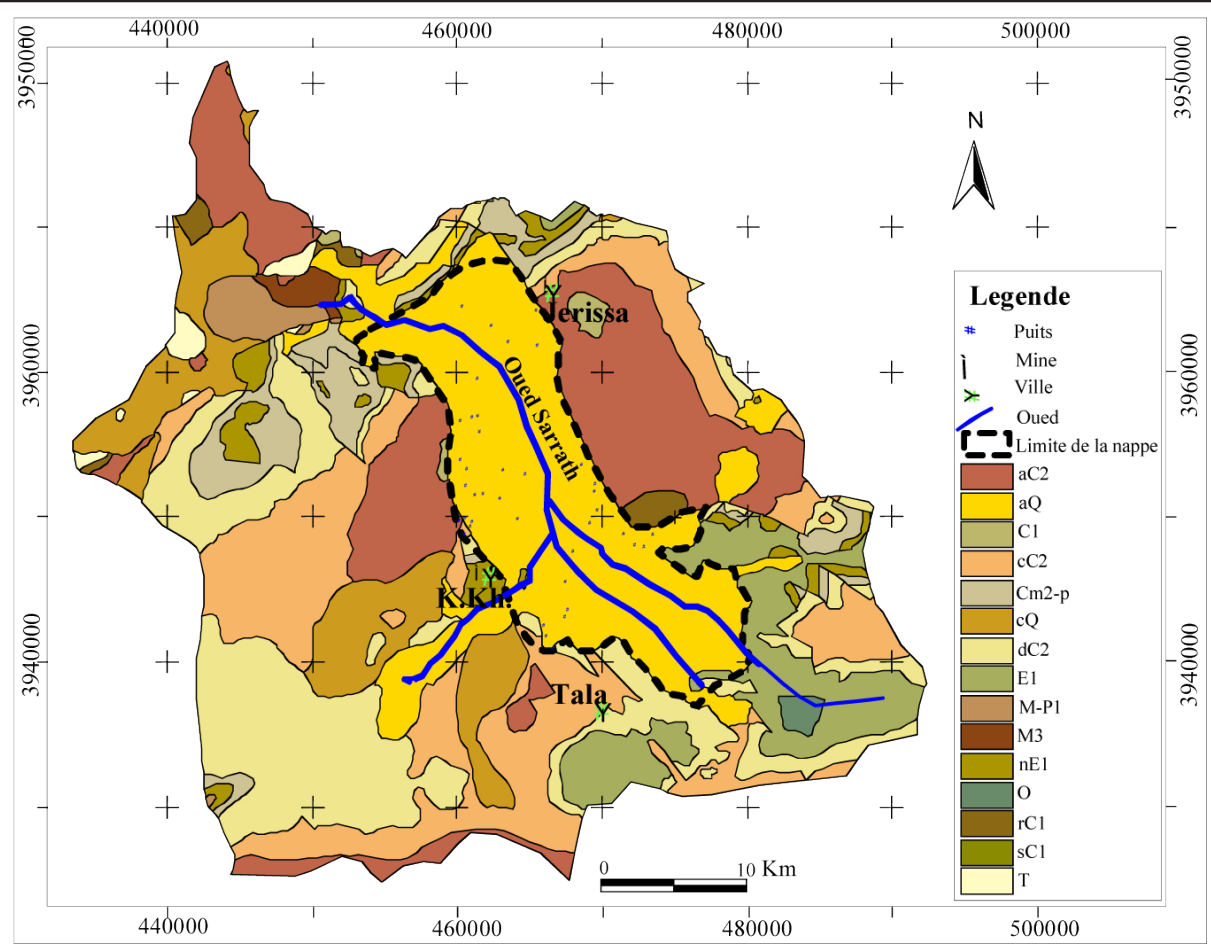

Figure 1. Carte géologique et emplacement des puits échantillonnés dans la nappe phréatique de K. Khasba (carte géologique de la Tunisie 1/500000).

Geologic map and the location of the studied mines and the sampling wells (geologic map of Tunisia 1/500000).

\section{MATÉRIEL ET MÉTHODES}

Pour évaluer l'impact des différentes sources de pollution sur les eaux de la nappe phréatique de K. Kh., nous avons procédé à un échantillonnage et à une analyse des terrils miniers, du sol situé dans la zone de recharge du côté de la mine de Jerissa et des eaux de la nappe phréatique.

\subsection{Mine de fer de Jérissa}

La mine de fer de Jérissa se situe au nord de la zone d'étude (Figure 1). Son exploitation remonte au début du XIX $\mathrm{X}^{\mathrm{e}}$ siècle. La minéralisation est constituée de sidérite, partiellement oxydée est surtout stratiforme. En 2007, les réserves ont été estimées à 90 millions de tonnes d'hématite $\left(\mathrm{Fe}_{2} \mathrm{O}_{3}\right)$ et 15 millions de tonnes de sidérite $\left(\mathrm{Fe}_{2} \mathrm{CO}_{3}\right)$, en plus de quelques tonnes de magnétite $\left(\mathrm{Fe}_{3} \mathrm{O}_{4}\right)$ et de pyrite $\left(\mathrm{FeS}_{2}\right)$. Autour de la mine, on distingue de grandes quantités de stériles qui risquent d'être dispersés en aval sous l'action des facteurs naturels et anthropiques. Pour quantifier les métaux dans le terril, six échantillons ( $\mathrm{Jr} 0-\mathrm{Jr} 5)$ ont été prélevés à différents endroits.

\subsection{Mine de phosphates de Kalaa Khasba}

La mine des phosphates de K. Kh. qui a fonctionné de 1893 jusqu'en 1993, a engendré des centaines de milliers de mètres cubes de terrils riches en métaux toxiques (MLAYAH et al., 2009; ZAIER, 1999). Plusieurs études ont montré que les mines de phosphates en Tunisie, comme dans le reste du monde, présentent des teneurs élevées en métaux (ANOUAR et al., 2006; BEJI SASSI et SASSI, 1999; GNANDI et al., 2006; TLIG et al., 1987). Dans le but de déterminer les éléments indésirables qui peuvent détériorer la qualité de l'eau, sept échantillons représentatifs ont été prélevés au niveau du terril de la mine de K. Kh (KK1-KK7).

\subsection{Sol autour de la mine de fer de Jérissa}

Le sol limitrophe de la mine de K. Kh. est bien protégé contre la propagation des rejets par voie hydrique par une digue protectrice. Cependant, le sol autour du terril de la mine de Jérissa est exposé aux différents vecteurs de transport. Ainsi, il a été échantillonné suivant trois radiales, jusqu'à une distance d'environ $1500 \mathrm{~m}$. Au total, 21 échantillons de sols ont été collectés à l'aval du terril (Figure 2).

Les échantillons solides (terrils et sols) ont été séchés à l'air libre, puis homogénéisés et subdivisés par quartage. Le broyage a été réalisé à l'aide d'un mortier en agate. Cinq cent grammes de chaque échantillon du sol ont été soumis à une séparation granulométrique à sec pour récupérer les refus 


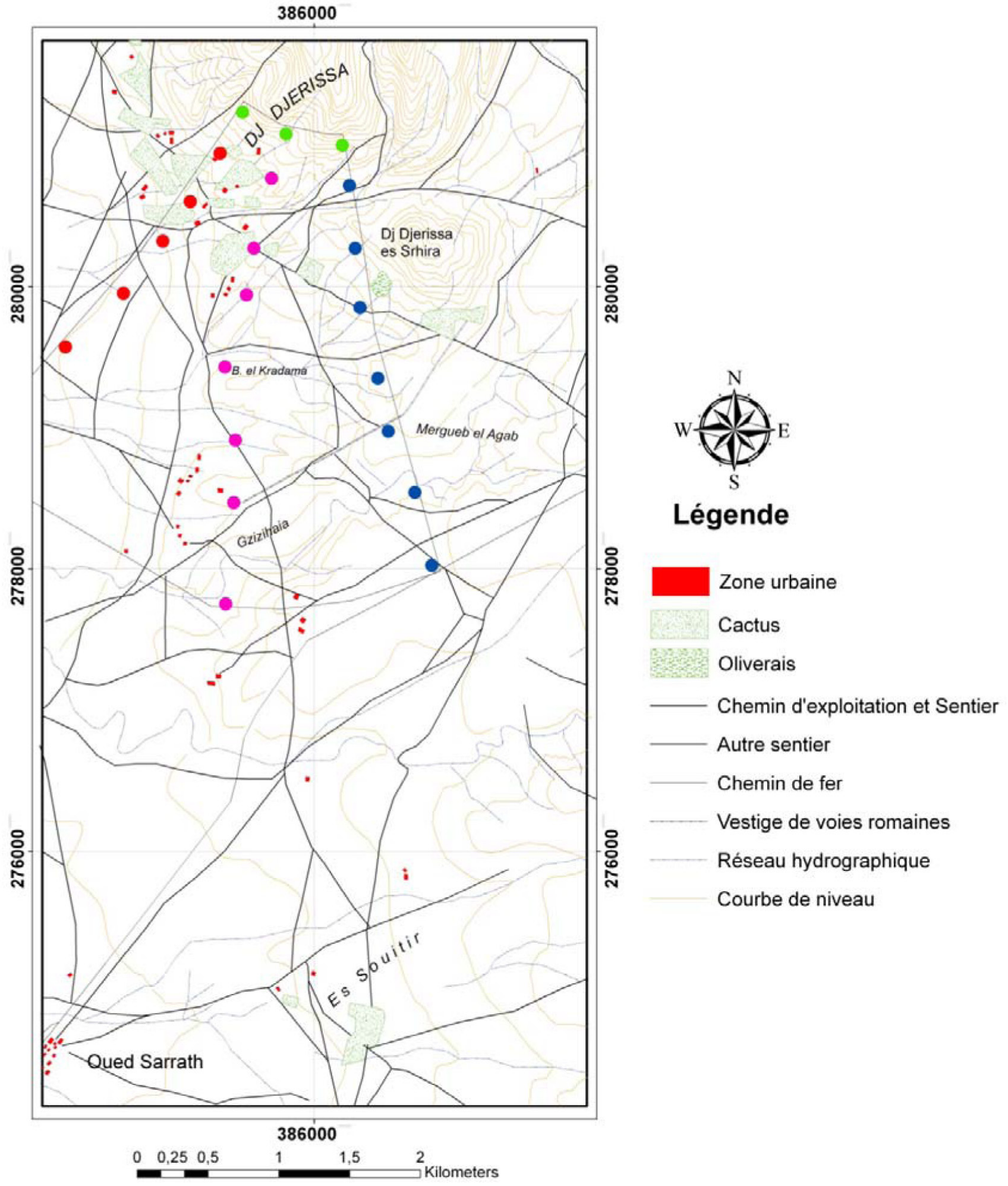

Figure 2. Emplacement des échantillons du sol aux environs de la mine de Jérissa. Soil sampling locations near the Jerissa mine. 
$(\varnothing<2 ; 2<\varnothing<5$ et $\varnothing>5 \mathrm{~mm})$ dans le but d'évaluer la concentration en métaux de chaque fraction.

\subsection{Eaux de la nappe souterraine}

Dans cette étude, une cinquantaine de puits, assez bien répartis sur toute la plaine, ont été échantillonnés après un pompage de 15 minutes (Figure 1). L'emplacement des puits a été déterminé à l'aide d'un GPS de type Garmin. Le niveau statique a été mesuré grâce à une sonde piézométrique. Les échantillons d'eau ont été prélevés dans des bouteilles en polyéthylène, préalablement lavées avec une solution alcaline et rincées à l'eau bidistillée, puis transportées au laboratoire à $4{ }^{\circ} \mathrm{C}$. Plusieurs paramètres physico-chimiques ont été déterminés in situ, à savoir : la température, la conductivité électrique (CE) et le $\mathrm{pH}$.

Au laboratoire, les eaux sont filtrées à l'aide des filtres $45 \mu \mathrm{m}$. Vingt échantillons d'eau correspondant aux puits situés en amont des deux principales sources de pollution ont été choisis pour analyse totale par ICP/MS, aux laboratoires (ACME Analytical Laboratories LTD, Vancouver, Canada), dans le but de déterminer les teneurs des métaux, à savoir : $\mathrm{Al}, \mathrm{As}, \mathrm{B}, \mathrm{Ba}, \mathrm{Br}, \mathrm{Cd}, \mathrm{Co}, \mathrm{Cr}, \mathrm{Cu}, \mathrm{Hg}, \mathrm{Mo}, \mathrm{Ni}, \mathrm{Sr}, \mathrm{U}$ et $\mathrm{Zn}$. L'erreur relative en déterminant le $\mathrm{Mo}, \mathrm{Co}, \mathrm{Cd}, \mathrm{Sb}, \mathrm{Bi}, \mathrm{U}$ et $\mathrm{B}$, est estimée à environ $2 \%$. Elle est de l'ordre de $10 \%$ pour le $\mathrm{Cu}, \mathrm{Pb}, \mathrm{Zn}, \mathrm{Ni}, \mathrm{Mn}, \mathrm{As}, \mathrm{V}$, La et $\mathrm{Cr}$.

Les orthophosphates $\left(\mathrm{PO}_{4}^{3-}\right)$ et les éléments azotés $\left(\mathrm{NH}_{4}^{+}\right.$, $\mathrm{NO}_{2}{ }^{-}$et $\mathrm{NO}_{3}{ }^{-}$) dans les échantillons d'eau ont été déterminés par la méthode colorimétrique décrite par RODIER et al. (1996), à l'aide d'un spectromètre Perkin Elmer (Lambda 25 UV/ IS Spectrometer). Les chlorures et les sulfates sont analysés à l'aide des électrodes spécifiques (Metrohm 692 pH/ Ion Metr). Le dosage des carbonates est effectué par une méthode volumétrique. La concentration des éléments majeurs dans le reste des puits $(\mathrm{Ca}, \mathrm{Mg}, \mathrm{Na}$ et $\mathrm{k})$ a été réalisée par un spectroflamme d'absorption atomique Perkin Elmer (AAnalyst 200).

Étant donné que la structure minéralogique des métaux présents dans les terrils et le sol est déterminante pour leur dissolution et la libération des métaux, en particulier la présence des sulfures, nous avons réalisé une analyse minéralogique des échantillons des terrils et du sol par un diffractomètre de RX (Philips PW1130/90), à l'Université d'Aveiro (Portugal).

\section{RÉSULTATS ET INTERPRÉTATIONS}

\subsection{Analyse minéralogique}

La composition minéralogique du terril et des sols aux environs de la mine de Jérissa est relativement semblable.
Les principaux minéraux présents dans ces échantillons sont : ankérite $\mathrm{Ca}(\mathrm{Mg}, \mathrm{Fe})\left(\mathrm{CO}_{3}\right)_{2}$, quartz $\left(\mathrm{SiO}_{2}\right)$, calcite $\left(\mathrm{CaCO}_{3}\right)$, hématite $\mathrm{Fe}_{2} \mathrm{O}_{3}$, goethite $\mathrm{FeO}(\mathrm{OH})$, sidérite $\left(\mathrm{Fe}_{2} \mathrm{CO}_{3}\right)$, pyrite $\left(\mathrm{FeS}_{2}\right)$, kaolinite et illite. Toutefois, nous pouvons également y trouver : limonite $\mathrm{FeO}(\mathrm{OH})$; galène $(\mathrm{PbS})$, cérusite $\left(\mathrm{PbCO}_{3}\right)$, sphalérite $(\mathrm{ZnS})$, pyrolusite $\left(\mathrm{MnO}_{2}\right)$, arsénopyrite (FeAsS), dialogite; chalcopyrite $\left(\mathrm{CuFeS}_{2}\right)$, chalchosine, malachite $\mathrm{Cu}_{2} \mathrm{CO}_{3}(\mathrm{OH})_{2}$, azurite $\mathrm{Cu}\left(\mathrm{CO}_{3}\right)_{2}(\mathrm{OH})_{2}$, pyrolusite $\left(\mathrm{MnO}_{2}\right)$ et calamine (AISSAOUI-ADJALI et al., 1988; MLAYAH et al., 2009).

La minéralogie des échantillons en provenance des terrils de la mine de phosphates de K. Kh. est assez hétérogène. Elle présente une prédominance de calcite, de quartz, d'apatite, de feldspaths et de dolomite. Des études antérieures ont mis en évidence la présence des phases accessoires telles que galène, plumbogummite, gypse, anhydrite, alunite $\left[\mathrm{KAl}_{3}\left(\mathrm{SO}_{4}\right)_{2}(\mathrm{OH})_{6}\right]$, jarosite $\left[\mathrm{KFe}_{3}\left(\mathrm{SO}_{4}\right)_{2}(\mathrm{OH})_{6}\right]$, thenardite $\left[\mathrm{Na}_{2} \mathrm{SO}_{4}\right]$, sidérite, pyrrhotite et également les phosphates et la sphalérite (MLAYAH et al., 2009; ZAIER, 1999).

L'exposition de ces terrils aux conditions atmosphériques engendre l'acidification des écoulements issus de ces rejets et, par la suite, l'enrichissement du sol avoisinant en sulfates et en métaux (BOBOS et al., 2006; MLAYAH et al., 2007, 2009).

\subsection{Analyse chimique}

\subsubsection{Terrils miniers et sols}

Les résultats d'analyses chimiques des échantillons des terrils sont inscrits dans le tableau 1. À partir de ce tableau, il s'avère que les terrils phosphatés sont riches en Mo (10-35 ppm), $\mathrm{Cu}$ (14-54 ppm), Ni (48-101 ppm), Cd (32-72 ppm), Cr (215-320 ppm), U (50-127 ppm), Sr (1,28-1,93 \%), $\mathrm{Zn}$ (160-336 ppm) et, évidemment, le phosphore avec des teneurs qui atteignent $20 \%$ de $\mathrm{P}_{2} \mathrm{O}_{5}$. Le P est un élément dominant dans les apatites, tandis que les autres métaux sont des oligoéléments indésirables, mais communs aux phosphates sédimentaires en Tunisie dont leurs teneurs varient d'une couche à l'autre et selon les régions (BEJI SASSI et SASSI, 1999; TLIG et al., 1987; ZAIER, 1999).

Les stériles de la mine de fer de Jérissa sont riches en Mn (2,5-4,6 \%), Pb (188-281 ppm), Ba (16-112 ppm), Zn (115-214 ppm), Sr (112-242 ppm), Ni (28-39 ppm), Co (14-73 ppm) et enfin As (18-32 ppm). Les teneurs en fer varient entre $7,13 \%$ et $13 \%$. Leur dispersion en aval entraîne la pollution des sols avoisinants par les métaux toxiques. En effet, les oxyhydroxides de fer et manganèse piègent des quantités non négligeables d'éléments traces métalliques (COVELO et al., 2004; HALIM et al., 2009). Une corrélation 
Tableau 1. Concentrations de quelques éléments (ppm) présents dans les terrils (Fe et $\mathrm{S}$ en $\%, \mathrm{Hg}$ en ppb).

Table 1. Heavy metal concentrations ( $p \mathrm{pm}$ ) in the tailings (Fe, S in \%; $\mathrm{Hg}$ in ppb).

\begin{tabular}{cccccccccccccccc}
\hline & $\mathrm{Mo}$ & $\mathrm{Cu}$ & $\mathrm{Pb}$ & $\mathrm{Zn}$ & $\mathrm{Ni}$ & $\mathrm{Co}$ & $\mathrm{Mn}$ & $\mathrm{Fe}$ & $\mathrm{As}$ & $\mathrm{Sr}$ & $\mathrm{Cd}$ & $\mathrm{Cr}$ & $\mathrm{Ba}$ & $\mathrm{S}$ & $\mathrm{Hg}$ \\
\hline $\mathrm{KK} 1$ & 14,1 & 14,4 & 2,9 & 229 & 38 & 0,5 & 57 & 0,64 & 3 & 1419 & 49,2 & 255 & 76 & 0,8 & - \\
$\mathrm{KK} 2$ & 15,3 & 30,2 & 2,2 & 247 & 57,1 & 0,4 & 61 & 0,29 & 2 & 1604 & 45,9 & 260 & 63 & 1,1 & - \\
$\mathrm{KK} 2$ & 14,1 & 26,2 & 2,2 & 257 & 61,6 & 0,6 & 63 & 0,3 & 2 & 1574 & 51,7 & 254 & 64 & 1,1 & - \\
$\mathrm{KK} 4$ & 17,1 & 46,3 & 2,6 & 336 & 101 & 0,6 & 24 & 0,26 & - & 1936 & 72,2 & 319 & 32 & 1,1 & - \\
$\mathrm{KK} 5$ & 9,9 & 54 & 3,3 & 302 & 48,8 & 0,5 & 184 & 0,16 & 4,6 & 1345,7 & 44,18 & 251 & 100 & 0,71 & - \\
$\mathrm{KK6}$ & 12,8 & 30,3 & 11,7 & 285 & 65,2 & 0,7 & 60 & 0,26 & 4,6 & 1823 & 42,7 & 303 & 74 & 1,1 & 0,72 \\
$\mathrm{KK} 7$ & 34,7 & 18,1 & 21,3 & 255 & 50,9 & 3,7 & 133 & 1,23 & 5,7 & 1282 & 31,7 & 276 & 52 & 3,1 & 34,7 \\
$\mathrm{Jr} 1$ & 1,28 & 116 & 253 & 179 & 27,9 & 13,9 & 2517 & 7,13 & 26,8 & 242 & 0,35 & 14,6 & 103 & 0,06 & 471 \\
$\mathrm{Jr2}$ & 0,81 & 21,3 & 188 & 115 & 39 & 21,6 & 3747 & 9,02 & 27,7 & 111,9 & 0,24 & 10,4 & 16 & 0,14 & 405 \\
$\mathrm{Jr3}$ & 2,4 & 129 & 281 & 214 & 31 & 73 & 4631 & 10,1 & 32 & 231 & 0,31 & 15 & 112 & 0,15 & 350 \\
$\mathrm{Jr} 4$ & 1,6 & 87 & 179 & 123 & 36,3 & 31,2 & 3251 & 11,5 & 42 & 1325 & 0,3 & 80,8 & 228 & 0,2 & 330 \\
$\mathrm{Jr} 5$ & 1,1 & 65,2 & 156 & 177 & 28,9 & 22,4 & 4039 & 12,8 & 22 & 1456 & 0,42 & 28 & 273 & 0,4 & 130 \\
\hline
\end{tabular}

entre les différents éléments analysés dans le terril de la mine de Jérissa a été définie. Cette corrélation montre des valeurs importantes dans les terrils $[(\mathrm{Cu} / \mathrm{Co}=0,6 ; \mathrm{Zn} / \mathrm{Ni}=0,8$; $\mathrm{Zn} / \mathrm{Mn}=0,6 ; \mathrm{Zn} / \mathrm{Fe}=0,5 ; \mathrm{Zn} / \mathrm{Ba}=0,7 ; \mathrm{Ni} / \mathrm{Mn}=0,5 ;$ $\mathrm{Zn} / \mathrm{Sr}=0,73 ; \mathrm{Zn} / \mathrm{Cr}=0,4 ; \mathrm{Zn} / \mathrm{Al}=0,5 ; \mathrm{Ni} / \mathrm{Co}=0,6 ;$ $\mathrm{Ni} / \mathrm{Sr}=0,7 ; \mathrm{Mn} / \mathrm{Fe}=0,9 ; \mathrm{Mn} / \mathrm{Sr}=0,8 ; \quad \mathrm{Fe} / \mathrm{As}=0,4 ;$ $\mathrm{Ni} / \mathrm{Al}=0,7 ; \mathrm{Fe} / \mathrm{Sr}=0,7 ; \mathrm{As} / \mathrm{Cr}=0,4 ; \mathrm{Ni} / \mathrm{Ba}=0,7 ; \mathrm{As} / \mathrm{Al}=0,3 ;$ $\mathrm{Cr} / \mathrm{Al}=0,6 ; \mathrm{Ba} / \mathrm{Al}=0,5 ; \mathrm{Ni} / \mathrm{Cr}=0,6]$.

Les échantillons du sol, situés en pleine zone de recharge et à différentes distances du terril, font l'objet de cette étude. La moyenne des résultas d'analyses des fractions granulométriques de ces échantillons est donnée dans le tableau 2.

L'examen de ce tableau montre que la totalité des échantillons du sol présente une fraction fine $(<2 \mathrm{~mm})$ dominante (> $50 \%)$. La concentration en métaux toxiques s'avère très élevée, en particulier dans la fraction fine la plus remobilisable sous l'action hydrique et éolienne (Figure 3). L'analyse chimique de ces échantillons montre qu'ils sont riches en $\mathrm{Mn}, \mathrm{Sr}, \mathrm{Ba}, \mathrm{Zn}, \mathrm{Cr}, \mathrm{Pb}, \mathrm{Ni}, \mathrm{Cu}$, As et Co.

L'indice de pollution $\left(\mathrm{P}_{\mathrm{i}}\right)$, défini comme étant le rapport de la concentration d'un élément (i) dans le sédiment $\left(\mathrm{C}_{\mathrm{i}}\right)$ sur la concentration du fond géochimique $\left(\mathrm{S}_{\mathrm{i}}\right): \mathrm{P}_{\mathrm{i}}=\frac{\mathrm{C}_{\mathrm{i}}}{\mathrm{S}_{\mathrm{i}}}$, nous a permis de démonter que les valeurs les plus élevées ont été enregistrées pour l'As et $\mathrm{Pb}$ (30 et 25). Pour le $\mathrm{Cu}$ et $\mathrm{Zn}$, les indices de pollution sont estimés à 20 et 11 respectivement. L'ordre de contamination déterminé est : $\mathrm{As}>\mathrm{Pb}>\mathrm{Cu}>$ Zn. Cette contamination est attribuée aux effets conjugués des agents de transport hydrique et éolien qui peuvent drainer la fraction fine à des distances très importantes et son accumulation dans les dépressions qui constituent la zone de recharge de la nappe.

\subsubsection{Résultats d'analyse des eaux}

\subsubsection{Paramètres hydrologiques et physico-chimiques}

Les différentes mesures des niveaux statiques de la nappe phréatique et des altitudes des puits nous ont permis d'établir la carte piézométrique de la nappe (Figure 4). À la lumière de cette carte, nous pouvons distinguer deux grandes directions d'écoulement à l'amont. La première direction concerne la rive gauche de Serrat, où l'eau s'écoule du Sud-Ouest vers le NordEst. Le gradient hydraulique moyen dans cette zone est de l'ordre de 1,9\%. La seconde, drainée par l'oued Sekka, prend la direction du Sud-Est vers le Nord-Ouest, avec un gradient de l'ordre de $0,9 \%$. Ces directions convergent à l'aval de la nappe au niveau de l'oued Sarrat, qui constitue l'exutoire de cette nappe, avec un gradient hydraulique largement plus faible (moins de 0,2\%). Ceci laisserait penser que la vitesse d'écoulement de l'eau serait beaucoup plus faible à l'aval qu'à l'amont de la nappe, ce qui se traduirait par des temps de séjour plus importants en aval.

\subsubsection{Qualité physico-chimique}

\section{Conductivité électrique}

La répartition spatiale de la conductivité électrique (CE) montre que cette superficie croît dans le même sens que l'écoulement souterrain (Figure 5). Les valeurs les plus faibles sont enregistrées en amont de la plaine avec une salinité inférieure à $1 \mathrm{~g} \bullet \mathrm{L}^{-1}$, notamment au niveau des zones de recharge situées au Sud-Est et au Sud-Ouest de la nappe. Cependant, les valeurs les plus élevées sont mesurées au niveau de la terminaison de Jbel el Hout, où l'oued Serrath devient l'exutoire de cette 
Tableau 2. Concentration des métaux (ppm, Fe \%) dans les fractions granulométriques du sol.

Table 2. Heavy metals concentrations (ppm, $\mathrm{Fe} \%$ ) in the soil size fractions.

\begin{tabular}{|c|c|c|c|c|c|c|c|c|c|c|c|c|c|c|c|c|}
\hline & $\varnothing \mathrm{mm}$ & $\%$ pondéral & Mo & $\mathrm{Cu}$ & $\mathrm{Pb}$ & $\mathrm{Zn}$ & $\mathrm{Ni}$ & Co & $\mathrm{Mn}$ & $\mathrm{Fe}$ & As & $\mathrm{U}$ & $\mathrm{Sr}$ & $\mathrm{Cd}$ & $\mathrm{Cr}$ & $\mathrm{Ba}$ \\
\hline \multirow{3}{*}{ So } & $\varnothing>5$ & 26,8 & 1,2 & 21,3 & 41 & 124 & 22,6 & 7,4 & 598 & 3,02 & 9 & 2,2 & 531 & 0,4 & 60,8 & 181 \\
\hline & $2>\varnothing>5$ & 20 & 1,4 & 21,2 & 49,4 & 115 & 23,9 & 7,8 & 593 & 2,71 & 7 & 1,9 & 482 & 0,3 & 38,6 & 180 \\
\hline & $\varnothing<2$ & 65,7 & 1,4 & 22,2 & 46,7 & 119 & 25,7 & 8,4 & 679 & 3,72 & 7 & 2,1 & 497 & 0,4 & 39,8 & 178 \\
\hline \multirow{3}{*}{ S1 } & $\varnothing>5$ & 16 & 1,3 & 25,5 & 39,2 & 93 & 22,6 & 8,9 & 737 & 3,22 & 12 & 2,1 & 499 & 0,4 & 53 & 179 \\
\hline & $2>\varnothing>5$ & 16,6 & 1,2 & 82,2 & 50,9 & 103 & 28,5 & 13,2 & 757 & 3,57 & 9 & 2,2 & 523 & 0,3 & 53,8 & 178 \\
\hline & $\varnothing<2$ & 67,2 & 1,2 & 32,6 & 38,9 & 96 & 24,9 & 9,7 & 786 & 4,24 & 11 & 2,2 & 493 & 0,3 & 53,3 & 175 \\
\hline \multirow{3}{*}{ S2 } & $\varnothing>5$ & 21,6 & 1,5 & 23,4 & 41 & 127 & 24,6 & 10,2 & 701 & 3,07 & 10 & 2,7 & 574 & 0,3 & 51,1 & 201 \\
\hline & $2>\varnothing>5$ & 23 & 1,5 & 22,3 & 44,7 & 126 & 24,3 & 9,9 & 683 & 3,21 & 11 & 2,5 & 631 & 0,4 & 53,6 & 202 \\
\hline & $\varnothing<2$ & 55,4 & 1,6 & 23,9 & 44 & 125 & 27,5 & 8,1 & 799 & 3,13 & 10 & 2,5 & 562 & 0,3 & 58,3 & 202 \\
\hline \multirow{3}{*}{ S3 } & $\varnothing>5$ & 25,6 & 1,5 & 21 & 41,5 & 143 & 34,3 & 10,6 & 579 & 3,07 & 8 & 2,9 & 574 & 0,4 & 68,5 & 278 \\
\hline & $2>\varnothing>5$ & 24 & 1,4 & 19,2 & 47,4 & 162 & 34,1 & 10,5 & 560 & 3,14 & 9 & 2,7 & 576 & 0,4 & 66,5 & 460 \\
\hline & $\varnothing<2$ & 50,4 & 1,5 & 18,9 & 40,2 & 131 & 29,4 & 9,1 & 593 & 3,89 & 10 & 2,7 & 540 & 0,4 & 63,4 & 237 \\
\hline
\end{tabular}

a)

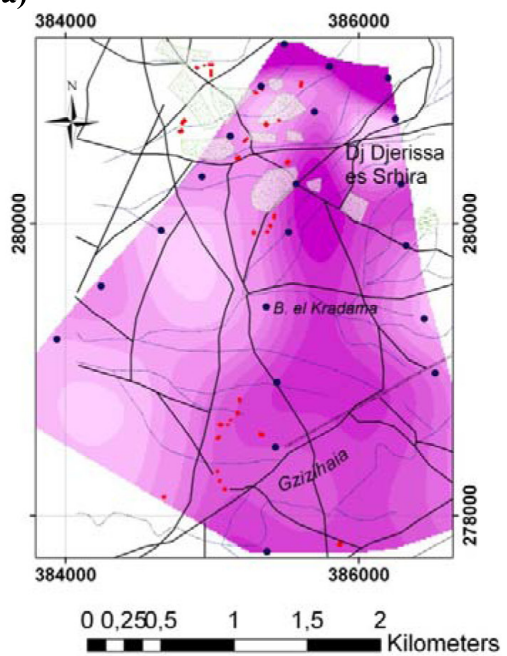

b) 384000

Teneur en As fraction $<2 \mathrm{~mm}$

Légende

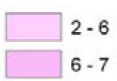

$\square-8$

$8-10$

$10-11$

$11-12$

$12-13$

$13-14$

$14-15$

$15-50$

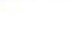

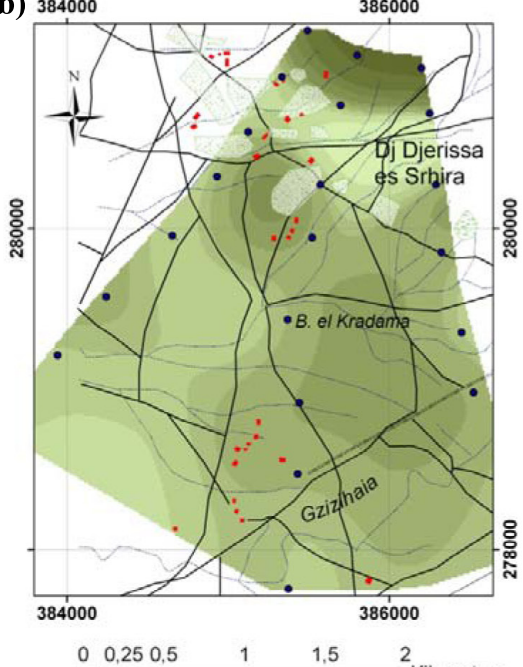

Teneur en $\mathrm{Cu}$ fraction $<2 \mathrm{~mm}$

Légende

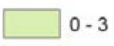

- $3-15$

$\square-22$

22- 26

$26-35$

$35-45$

$45-70$

$70-100$

$100-180$

$180-300$

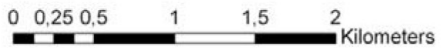

c)

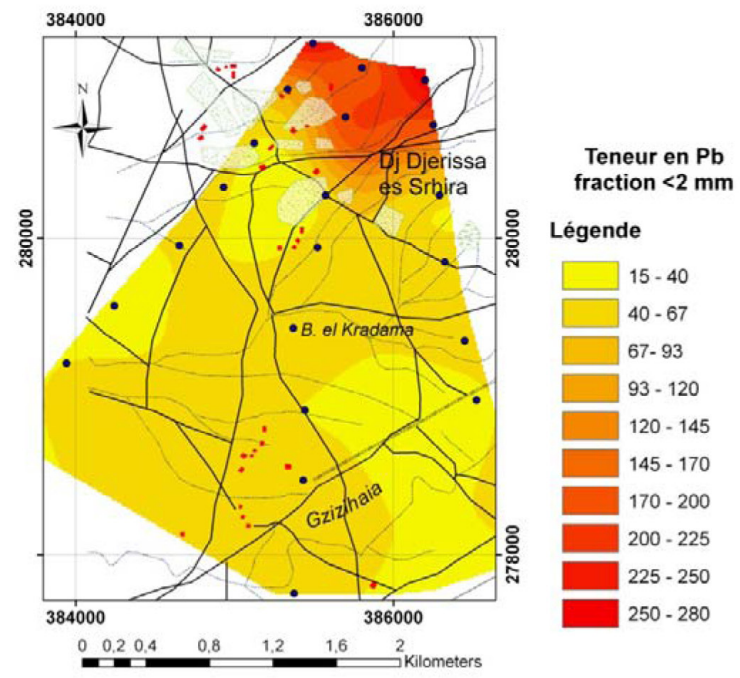

d)

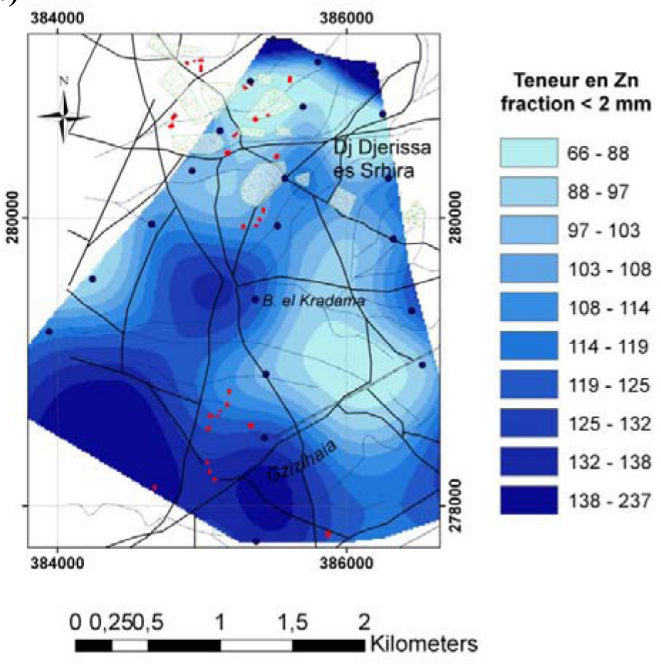

Figure 3. Cartes de répartition des métaux dans la fraction fine du sol : (a) As; (b) $\mathrm{Cu}$; (c) $\mathrm{Pb}$; (d) $\mathrm{Zn}$. Spatial distribution of metals in the fine grain size soil fractions (a): $A s ;(b): C u ;(c): P b ;(d): Z n$. 


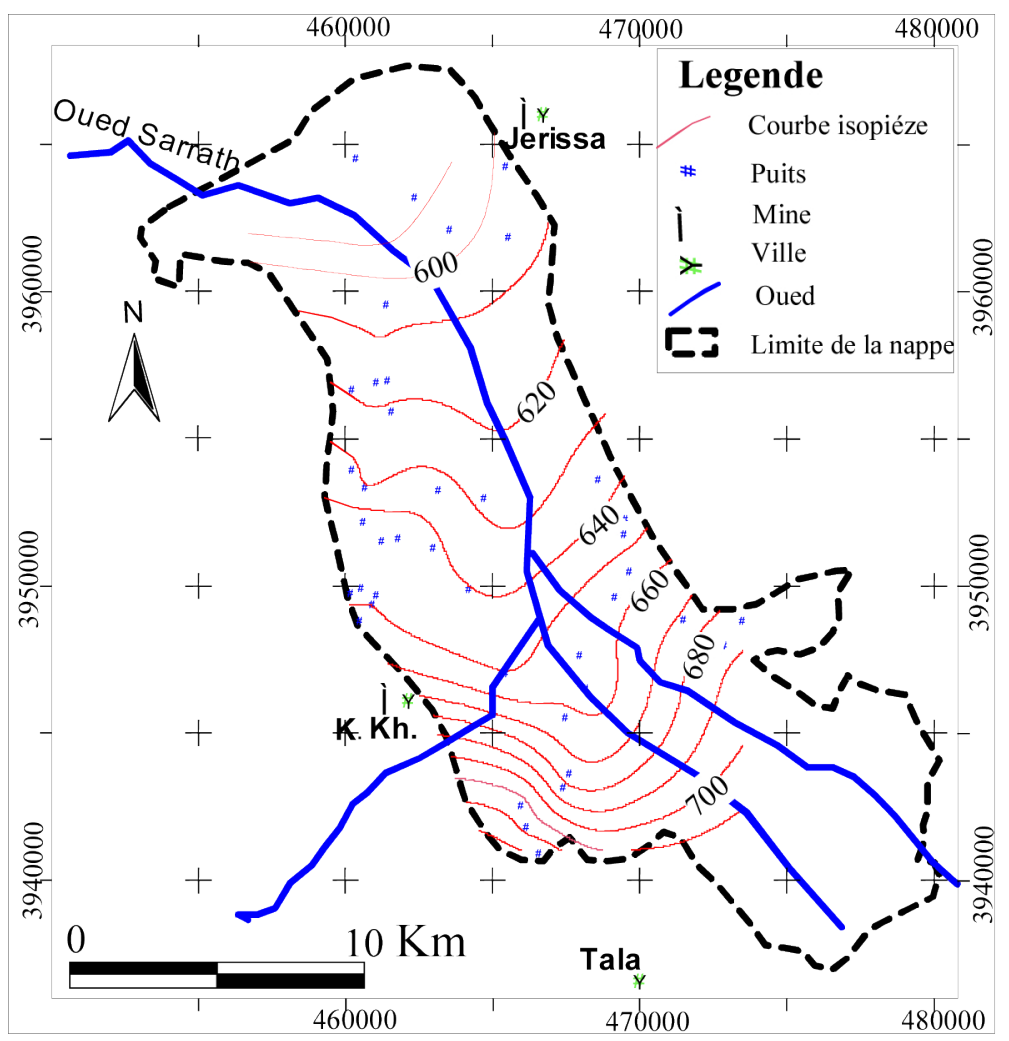

Figure 4. Carte piézométrique de la nappe phréatique de Kalaa Khasba (m). Piezometric map of Kalaa Khasba groundwater (m).

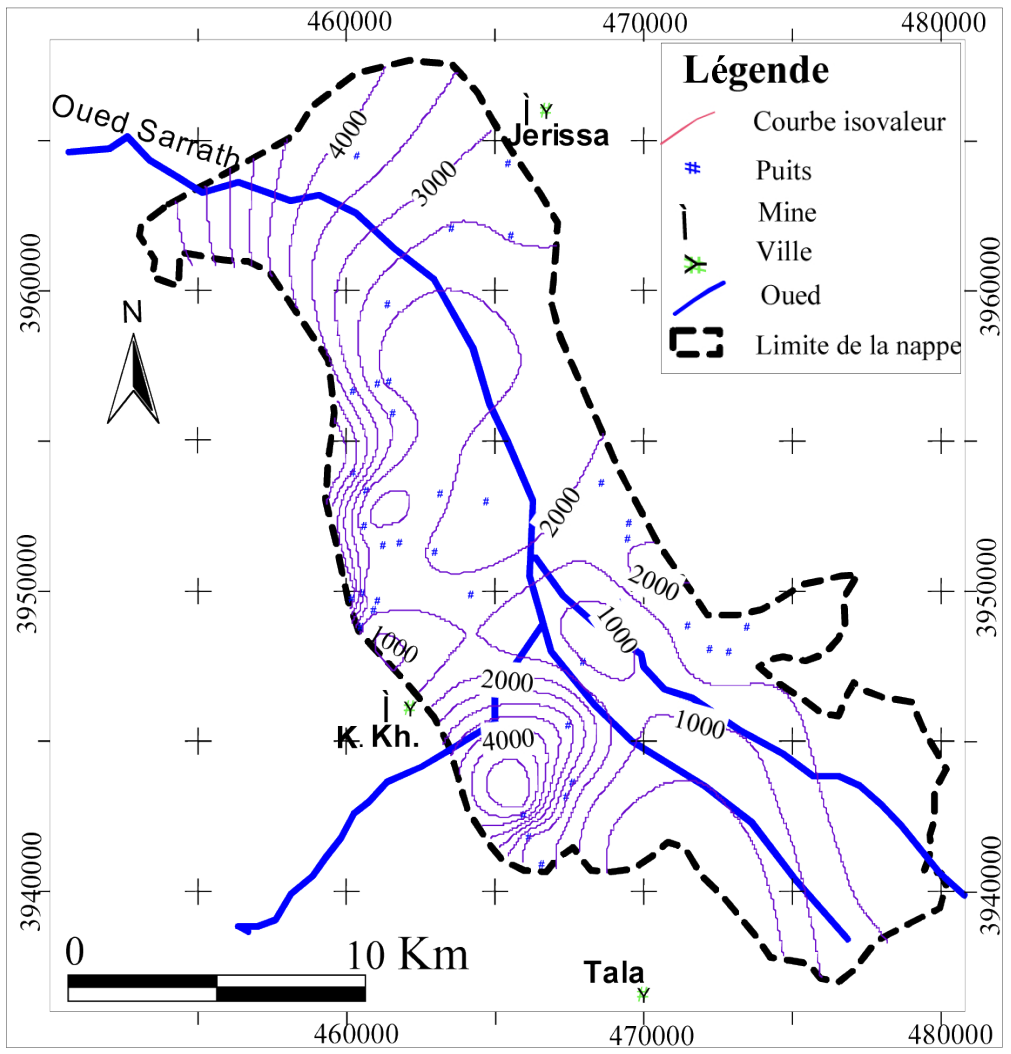

Figure 5. Carte de la conductivité électrique des eaux $\left(\mu s^{\bullet} \mathrm{cm}^{-1}\right)$ de la nappe phréatique de Kalaa Khasba.

Map of Kalaa Khasba groundwater electric conductivity $\left(\mu s^{\bullet} \mathrm{cm}^{-1}\right)$. 
nappe. La salinité en ces endroits atteint des valeurs de plus de $2 \mathrm{~g} \bullet \mathrm{L}^{-1}$. Cet enrichissement serait causé par le lessivage des couches géologiques alluviales relativement solubles. Le $\mathrm{pH}$ $(6,9-7,9)$ et la température $\left(20-23{ }^{\circ} \mathrm{C}\right)$ de ces eaux montrent souvent des valeurs caractérisant les eaux naturelles : ils restent stationnaires entre l'amont et l'aval du bassin.

\section{Géochimie des majeurs}

Les résultats d'analyse montrent que la concentration des cations majeurs varie d'un élément à l'autre. Les valeurs les plus élevées sont celles du $\mathrm{Na}^{+}\left(123-1840 \mathrm{mg} \bullet \mathrm{L}^{-1}\right)$ suivies du $\mathrm{Ca}^{2+}\left(10-343 \mathrm{mg} \bullet \mathrm{L}^{-1}\right)$ et $\mathrm{Mg}^{2+}\left(4-156 \mathrm{mg} \bullet \mathrm{L}^{-1}\right)$. Le $\mathrm{K}^{+}$montre des valeurs relativement faibles par rapport aux autres éléments $\left(2-65 \mathrm{mg} \cdot \mathrm{L}^{-1}\right)$.

En particulier, le cation principal $\left(\mathrm{Ca}^{2+}\right)$ doit souvent son origine à des carbonates tels que la calcite et la dolomite et des évaporites (gypse, $\left(\mathrm{CaSO}_{4}, 2 \mathrm{H}_{2} \mathrm{O}\right)$ ). Les principales sources du magnésium sont la biotite et la dolomite, tandis que le $\mathrm{K}^{+}$ est souvent libéré par des formations argileuses (HO, 2001). Des concentrations relativement élevées en $\mathrm{Na}^{+}$et en $\mathrm{Ca}^{2+}$, par rapport au $\mathrm{Mg}^{2+}$ et $\mathrm{K}^{+}$dans les eaux souterraines, suggèrent l'influence du phénomène de la dissolution élevée des roches sédimentaires (calcite et dolomite) et évaporitiques (halite et gypse). Notons que même si les engrais et les fertilisants contiennent également des quantités considérables en $\mathrm{K}^{+}$ et $\mathrm{Na}^{+}$, leurs concentrations mesurées sont celles du fond géochimique, même dans les zones où nous avons enregistré des teneurs élevées en nitrates. Il semblerait ainsi que la migration souterraine de ces éléments soit influencée par les phénomènes de sorption, exercés par divers composants du sol, tels que la matière organique et les argiles réputées par leur capacité adsorbante relativement élevée (BRIGATTI et al., 2000). Les principaux anions présents dans les eaux souterraines sont les $\mathrm{Cl}^{-}, \mathrm{SO}_{4}^{2-}, \mathrm{HCO}_{3}^{-}$et $\mathrm{NO}_{3}^{-}$. Il est important de souligner que la qualité des eaux souterraines montre une large variabilité dans la concentration des $\mathrm{Cl}^{-}\left(112-1029 \mathrm{mg}^{\bullet} \mathrm{L}^{-1}\right)$ et qui évoluent dans le même sens que la CE. En effet, la salinité, et en particulier les chlorures, montre une augmentation de l'amont vers l'aval. Les formes de chlorures ne sont pas complexées par les différents composants du sol et restent en solution. Ces anions sont habituellement utilisés comme des traceurs permettant d'identifier le sens d'écoulement souterrain (WEIL et al., 1990). Les concentrations de $\mathrm{SO}_{4}^{2-}\left(25-1173 \mathrm{mg} \bullet \mathrm{L}^{-1}\right)$ et $\mathrm{NO}_{3}^{-}\left(6-120 \mathrm{mg}^{\bullet} \mathrm{L}^{-1}\right)$ sont par endroits relativement élevées dans les échantillons d'eau de la nappe de K. Kh. Une telle tendance pourrait être attribuée, d'une part, au lessivage des formations gypseuses et, d'autre part, au déversement des eaux usées urbaines non traitées. Les concentrations des ions $\mathrm{HCO}_{3}$ dans les eaux souterraines varient entre 220 et $540 \mathrm{mg} \bullet \mathrm{L}^{-1}$. Les valeurs les plus élevées sont rencontrées au niveau des limites de l'aquifère. La présence de ces ions pourrait être due à la dissolution des minéraux carbonatés (carbonates et dolomites) qui délimitent la plaine et à la biodégradation de la matière organique (HALIM et al., 2009; HO, 2001).

Les analyses chimiques des eaux des différents puits, portées sur le diagramme de Piper, montrent que leurs faciès géochimiques présentent une évolution notable de l'amont à l'aval. Les eaux en amont de l'aquifère présentent un faciès carbonaté magnésien à calcique traduisant l'effet des roches calcaires et dolomitiques, ainsi que la circulation dans des séries grossières. Le passage de cette eau à travers des formations plus ou moins argileuses, riches en sulfates, lui fait acquérir un caractère plus sulfaté en conservant tout de même sa nature calcique ou magnésienne.

En traversant les couches plus riches en argiles et sous l'effet du phénomène de sorption, les concentrations des cations bivalents diminuent aux dépens du sodium et les eaux finissent par avoir un faciès chloruré sodique. Cette évolution au niveau des faciès est le résultat des réactions communes d'échange qui se font au cours de l'écoulement souterrain entre le $\mathrm{Ca}^{2+}, \mathrm{Mg}^{2+}$ en solution et le $\mathrm{Na}^{+}$, fortement absorbé par les argiles et la matière organique et où les ions bivalents tendent à remplacer les ions monovalents (APPELO et POSTMA, 1999). Cette réaction est réversible : un apport élevé d'ions monovalents peut remplacer les ions bivalents (FETTER, 1994).

Les ions monovalents et bivalents sont des indicateurs du temps de séjour des eaux souterraines. Un long temps de séjour se traduit généralement par des concentrations plus élevées en cations monovalents et une faible alcalinité au dépend des chlorures et sulfates. Les cartes des conductivités et des isopièzes (Figures 4 et 5) révèlent cette évolution géochimique des eaux : la partie amont, où les courbes de niveau sont serrées, se caractérise par un faciès géochimique carbonaté-calcique à magnésien. Au centre de la nappe, le temps de séjour deviendrait plus important, en traversant les couches à dominance argileuse riches en gypse, le faciès devient chloruré-sulfaté, calcique à sodique (BOUHLASSA ET AIACHI, 2004).

\section{Les sels nutritifs}

Les concentrations des orthophosphates dans les eaux de la nappe de K. Kh. sont relativement faibles et parfois inférieures à la limite de détection. Des résultats similaires ont été observés lors d'études sur des sites réels (KRAPAC et al., 2002; STÄPFLI et MADRAMOOTOO, 2006). Dans la zone d'étude, le phosphore est utilisé en agriculture sous forme de Triple Super Phosphate (TSP), Di Ammonium Phosphate (DAP), à raison de $100 \mathrm{~kg}^{\bullet} \mathrm{ha}^{-1} \bullet \mathrm{an}^{-1}$ et du fumier. Les faibles concentrations en orthophosphates dans les eaux souterraines sont dues au fait que cet élément est très fortement adsorbé par les constituants du sol, principalement par les oxydes de fer et d'aluminium (HALIM et al., 2009). Toutefois, il est important de souligner que le phosphore peut être relargué dans les eaux 
souterraines suite à la réduction des oxyhydroxydes de fer, sous l'effet des bactéries anaérobies (McARTHUR et al., 2004).

Dans les eaux naturelles, l'azote peut être sous plusieurs formes, soit des composés organiques complexes, soit sous forme minérale la plus simple $\left(\mathrm{NH}_{4}^{+}, \mathrm{NO}_{2}^{-}, \mathrm{NO}_{3}^{-}\right) \mathrm{n}$. Les paramètres physico-chimiques $\left(\mathrm{pH}, \mathrm{O}_{2}\right.$ dissous) influent la présence de l'une ou de l'autre forme (LANDREAU, 1990). Dans les eaux de surface ou souterraines, la forme la plus dominante est celle des nitrates.

L'analyse de l'eau de la nappe de K. Kh. a montré que plus de $90 \%$ des puits étudiés présentent des concentrations en $\mathrm{NH}_{4}^{+}$inférieures à la limite de détection. L'ammonium semble avoir le même comportement que le phosphore. Il semble s'adsorber à la surface du sol et dans les premiers centimètres par les constituants du sol pour former une réserve nutritive importante pour les plantes.

La concentration des nitrates dans les eaux étudiées montre des teneurs relativement importantes : $15 \%$ des puits excèdent les normes fixées par l'OMS (Organisation Mondiale de la Santé) pour les eaux potables (WHO, 2004). La concentration la plus importante $\left(130 \mathrm{mg} \cdot \mathrm{L}^{-1}\right)$ est observée au puit $\mathrm{n}^{\circ} 34$, le reste des puits montre des teneurs qui varient entre 6 et $120 \mathrm{mg} \bullet \mathrm{L}^{-1}$, dont $84 \%$ ont des concentrations supérieures à $10 \mathrm{mg} \bullet \mathrm{L}^{-1}$ (valeur fixée pour l'eau potable par l'Agence Américaine de la Protection de l'Environnement, USEPA, 1995). La carte de répartition des nitrates dans les eaux souterraines montre que les valeurs les plus élevées, supérieures à $10 \mathrm{mg} \bullet \mathrm{L}^{-1}$, coïncident avec les champs à cultures intenses et à proximité des grandes agglomérations : Tala, K. Kh. et, à moindre degrés, à Jérissa (Figure 6). En dehors de ces zones, les concentrations en $\mathrm{NO}_{3}$ sont faibles. Des observations similaires ont été signalées par MATICIC (1999) et COSTA et al. (2002).

Il est important de signaler que dans toute la zone d'étude, les agglomérations sont dépourvues d'un réseau d'assainissement. Ainsi, les eaux infiltrées des fosses septiques (puits perdus), riches en éléments azotés, peuvent constituer une autre source de contamination des eaux souterraines. En effet, les teneurs d'azote dans les excréments humains sont de l'ordre de $5 \mathrm{~kg} \bullet$ personne ${ }^{-1} \bullet \mathrm{an}^{-1}$. À raison de 20000 habitants (Hidra, Tala, K. Kh., Jérissa et la population rurale), environ 100 tonnes d'azote sont rejetées annuellement dans le bassin versant et finissent par rejoindre la nappe. Les eaux usées, à usage domestique, présentent également une source ponctuelle de pollution riche en sels nutritifs et en sulfates. Ces eaux sont rejetées directement dans les cours d'eau et font que la concentration des nitrates dans les eaux souterraines est importante aux environs des villes riveraines.

\section{Métaux lourds dans les eaux de la nappe}

Les résultats d'analyse des métaux dans les eaux des puits situés en amont de la nappe phréatique de K. Kh. sont décrits

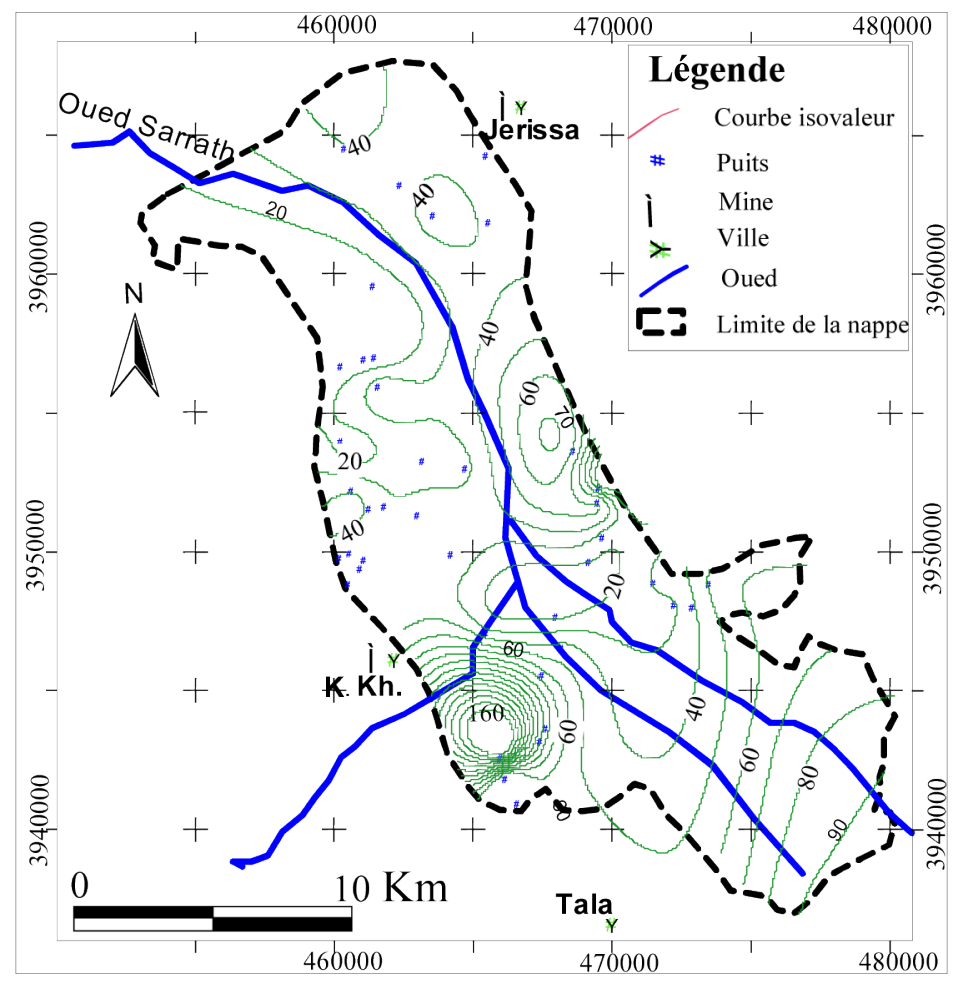

Figure 6. Carte de répartition des nitrates dans les eaux de la nappe phréatique de Kalaa Khasba $\left(\mathrm{mg}^{\bullet} \mathrm{L}^{-1}\right)$. Spatial distribution of nitrate concentrations in Kalaa Khasba groundwater $\left(m g \bullet L^{-1}\right)$. 
dans le tableau 3. L'examen des différentes concentrations montre qu'à l'exception du $\mathrm{B}$, où $100 \%$ des puits dépassent les normes de potabilité $(0,5 \mathrm{ppm})$, la majorité des éléments présente des teneurs inférieures aux valeurs tolérées pour l'eau potable, malgré leur abondance dans les rejets miniers et dans les sols (Tableaux 1 et 2). Les teneurs en $\mathrm{Hg}, \mathrm{Ni}, \mathrm{Cr}, \mathrm{Pb}$, et $\mathrm{Co}$ sont en dessous de la limite de détection pour respectivement $100 \%, 75 \%, 50 \%$ et $35 \%$ des puits étudiés. Toutefois, certains puits s'avèrent renfermer des concentrations non négligeables (puits $\mathrm{n}^{\text {os }} 1,6,10,11,14$ et 15) en métaux. L'échantillon 1, qui représente l'eau à la sortie de la galerie d'exploitation de la mine de Jérissa, a le $\mathrm{pH}$ le plus faible $(6,3)$ et la concentration la plus élevée en As $\left(3,8 \mu \mathrm{g} \bullet \mathrm{L}^{-1}\right)$.

La concentration du $\mathrm{Zn}$ dans les eaux de la nappe varie de 6 à $112 \mu \mathrm{g} \bullet \mathrm{L}^{-1}$. Les puits $\mathrm{n}^{\circ} \mathrm{s} 1,6$ et 10 présentent des teneurs supérieures à $40 \mu \mathrm{g} \bullet \mathrm{L}^{-1}$. Toutefois, ces valeurs demeurent en dessous des normes (Tableau 3). Dans les eaux souterraines, la principale source du $\mathrm{Zn}$ serait l'ajout des fertilisants (HALIM et al., 2009). Dans notre cas, nous ne pouvons pas négliger l'apport minier en $\mathrm{Zn}$. En effet, le rejet des mines renferme des quantités non négligeables (70-285 ppm), dont leur distribution par les différents agents de transport peut entraîner la pollution des eaux. Le $\mathrm{Zn}$ présente des corrélations négatives avec les cations majeurs ( $\mathrm{Ca}, \mathrm{Mg}, \mathrm{K}$ et $\mathrm{Na}$ ) et aussi avec le Fe et le Mn. Il sera possible que le Zn, avec sa mobilité, puisse avoir une multitude de sources dans les eaux de la nappe.
Le B est l'élément le plus abondant dans la nappe phréatique. Sa concentration est située entre 245 et $1400 \mu g^{\bullet} \mathrm{L}^{-1}$. Elle dépasse largement la concentration tolérée pour l'eau potable. Il présente des corrélations positives avec le sodium et le magnésium et négatives avec le calcium et le potassium (Figure 7). Le bore, présent en quantités très variables dans les roches éruptives et dans les argiles, peut être substitué avec le Si ou le C. Sa corrélation significative avec le sodium montre bien qu'il est adsorbé par les argiles et que sa substitution par le Ca est simultanée, ce qui se traduit par une corrélation négative avec cet élément (Figure 7).

Les teneurs en $\mathrm{Cu}$ varient de 1,3 à $4,3 \mu \mathrm{g} \bullet \mathrm{L}^{-1}$, la valeur la plus importante est observée dans les eaux de la galerie qui peut contaminer la nappe. L'origine de cet élément dans les eaux souterraines serait le résultat des applications agricoles (engrais, pesticides et autres dérivés cuivreux : DUMESTRE et al., 1999; KOMAREK et al., 2009; MICHAUD et al., 2007). La concentration du $\mathrm{Cu}$ dans l'eau est influencée par la taille des grains des engrais contenant cet élément, du temps de séjour et de l'humidité de sol (PETERSEN et al., 2004). Le Cu montre une corrélation positive avec les cations majeurs (Ca, $\mathrm{Na}, \mathrm{Mg}$ ), la valeur $\mathrm{R}^{2}$ de cette corrélation est pratiquement identique $(\approx 0,2)$ pour les trois cations (Figure 8$)$. Nous pouvons déduire que la concentration de cet élément dans les eaux de la nappe est liée au fond géochimique.

Tableau 3. Concentration (ppb) de quelques métaux analysés dans les eaux de la nappe de Kalaa Khasba.

Table 3. Heavy metal concentrations ( $p p b)$ in Kalaa Khasba groundwater.

\begin{tabular}{|c|c|c|c|c|c|c|c|c|c|c|c|c|c|c|}
\hline Puits & $\mathrm{Sr}$ & $\mathrm{Al}$ & As & B & $\mathrm{Ba}$ & $\mathrm{Br}$ & $\mathrm{Cd}$ & Co & $\mathrm{Cr}$ & $\mathrm{Cu}$ & $\mathrm{Hg}$ & Mo & $\mathrm{Ni}$ & $\mathrm{Zn}$ \\
\hline 1 & 3889 & 3 & 3,8 & 450 & 32,8 & 893 & nd & 0,02 & 15,1 & 4,3 & nd & 0,4 & 0,7 & 45 \\
\hline 2 & 3303 & 79 & 1,8 & 296 & 26,5 & 903 & 0,07 & 0,04 & 0,8 & 2,8 & nd & 2,3 & 0,6 & 7,3 \\
\hline 3 & 3503 & 58 & 1,5 & 283 & 25,6 & 881 & 0,07 & nd & nd & 1,5 & nd & 1,9 & nd & 29 \\
\hline 4 & 3865 & 77 & 2,2 & 272 & 30,2 & 1221 & 0,1 & 0,03 & 0,5 & 1,8 & nd & 2,5 & nd & 5,8 \\
\hline 5 & 2792 & 66 & 1,4 & 245 & 30,7 & 833 & nd & 0,04 & nd & 1,5 & nd & 2,6 & nd & 14,6 \\
\hline 6 & 6297 & 95 & 2,7 & 453 & 45,1 & 2262 & 4,6 & nd & nd & 4,1 & nd & 4,5 & nd & 51 \\
\hline 7 & 3318 & 120 & 2,3 & 300 & 29,8 & 1316 & nd & 0,03 & 1 & 1,5 & nd & 2 & nd & 35,6 \\
\hline 8 & 2766 & 82 & 1,9 & 386 & 19,8 & 1003 & nd & 0,03 & 1,9 & 1,3 & nd & 2,7 & nd & 21,9 \\
\hline 9 & 3144 & 112 & 1,9 & 364 & 27,4 & 1006 & nd & 0,03 & 1,2 & 1,6 & nd & 2,2 & nd & 14,4 \\
\hline 10 & 4170 & 57 & 2,9 & 552 & 29,2 & 1151 & 0,17 & 0,06 & nd & 2,3 & nd & 5 & nd & 112 \\
\hline 11 & 3196 & 74 & 1,9 & 449 & 18,3 & 982 & nd & 0,02 & 2 & 1,7 & nd & 2,8 & nd & 22,5 \\
\hline 12 & 3919 & 96 & 3,1 & 528 & 25,4 & 1257 & nd & 0,09 & 1,1 & 2 & nd & 3,6 & nd & 8,1 \\
\hline 13 & 4373 & 165 & 2,5 & 528 & 21,6 & 1347 & 0,06 & 0,05 & 1,6 & 2,5 & nd & 5,8 & 0,7 & 11 \\
\hline 14 & 2951 & 64 & 1,9 & 475 & 18,7 & 972 & nd & 0,04 & nd & 1,5 & nd & 4,8 & 0,3 & 17 \\
\hline 15 & 4783 & 3 & 3,6 & 373 & 21,6 & 1290 & nd & nd & nd & 2,6 & nd & 0,6 & 0,6 & 25,4 \\
\hline 16 & 6909 & 10 & 3,2 & 381 & 17,4 & 1066 & 0,09 & nd & 7 & 1,6 & nd & 0,5 & 0,4 & 9 \\
\hline 17 & 8835 & 57 & 1,3 & 912 & 16,3 & 572 & nd & 0,05 & nd & 2,3 & nd & 3,5 & nd & 17,7 \\
\hline 18 & 5458 & 67 & 2,2 & 571 & 16,6 & 1122 & nd & nd & nd & 1,5 & nd & 1,5 & nd & 10,2 \\
\hline 19 & 15368 & 71 & nd & 1155 & 14,9 & 1304 & nd & nd & nd & 2,5 & nd & 3,6 & nd & 22,2 \\
\hline 20 & 12053 & 38 & nd & 1400 & 14,8 & 1712 & nd & nd & nd & 2,5 & nd & 8,9 & nd & 24,7 \\
\hline Norme OMS & & 200 & 10 & 500 & 700 & - & 3 & - & 50 & 2000 & $<0,5$ & 70 & 70 & 3000 \\
\hline
\end{tabular}

nd : non détecté 
(a)

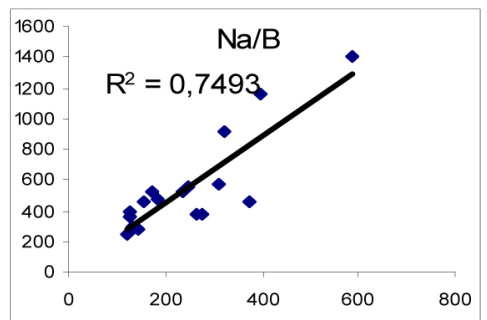

(b)

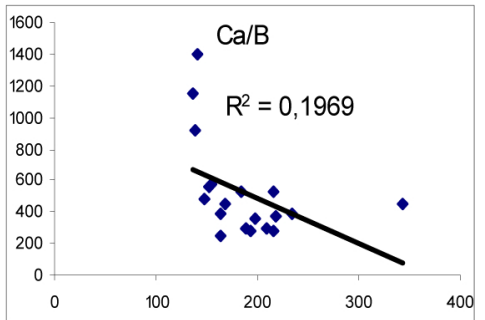

(c)

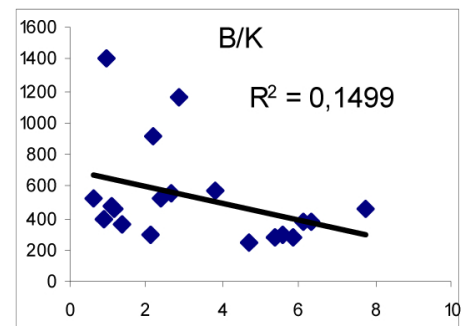

Figure 7. Corrélation du $B$ avec les éléments majeurs des eaux de la nappe $K$. Khasba : (a) : Na/B; (b) : Ca/B; (c) : B/K. Correlations between $B$ and major cations in Kalaa Khasba ground water: (a): Na/B; (b): Ca/b; (c): B/K.

(a)

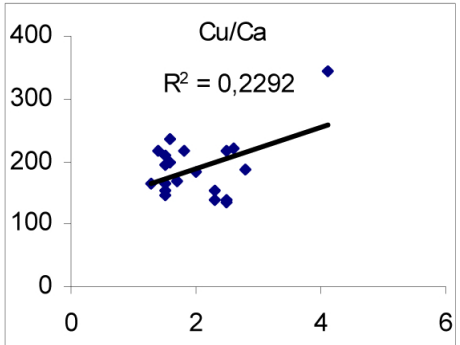

(d)

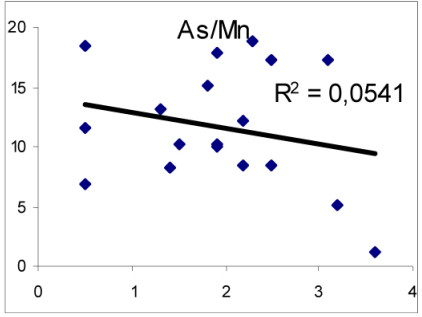

(g)

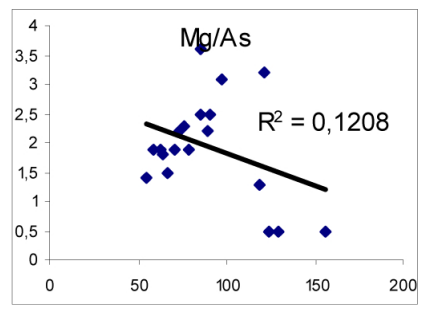

(j)

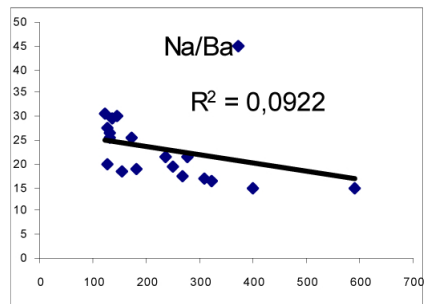

(b)

(c)

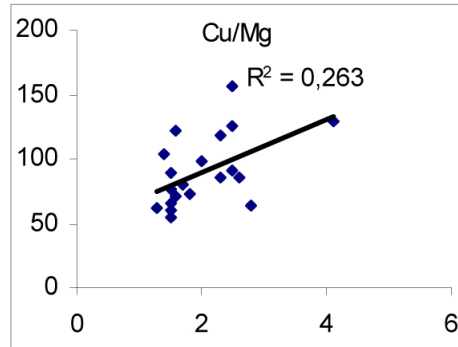

(e)

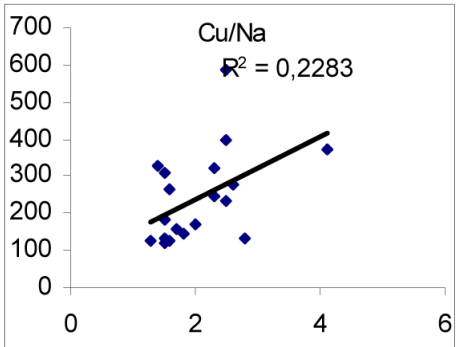

(f)

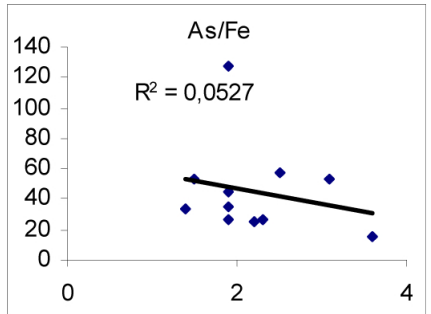

(h)

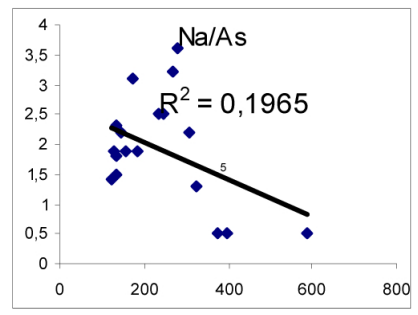

(i)

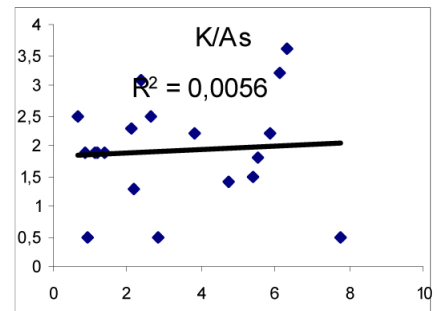

(k)

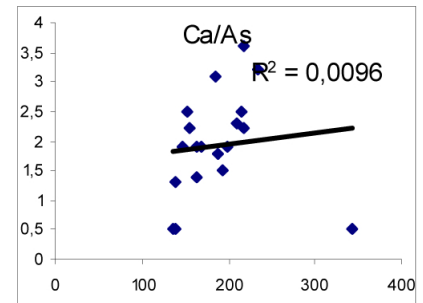

(I)

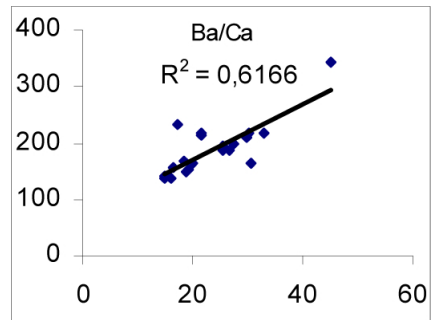

Figure 8. Corrélation du Cu (a; b; c), de l'As (d, e, f, g, h, i) et du Ba (j, k, l) avec les cations majeurs des eaux de la nappe de Kalaa Khasba.

Correlations between $C u(a ; b ; c), A s(d ; e ; f ; g ; b ; i), B a(j ; k ; l)$ and major cations in Kalaa Khasba ground water. 
La majorité des eaux présente des valeurs faibles en $\mathrm{Cd}$ : $65 \%$ des puits analysés montrent des concentrations inférieures à la limite de détection. Le puits $\mathrm{n}^{\circ} 6$ est le plus contaminé $\left(4,6 \mu \mathrm{g} \bullet \mathrm{L}^{-1}\right)$, suivi du puits $\mathrm{n}^{\circ} 10$ avec $0,17 \mu \mathrm{g}^{\bullet} \mathrm{L}^{-1}$. Pour le reste des puits, les teneurs ne dépassent guère $0,05 \mu \mathrm{g} \bullet \mathrm{L}^{-1}$. La détection de cet élément au niveau de la nappe ne pourrait pas provenir de la mine de Jérissa, étant donné qu'il existe à des teneurs négligeables $(0,4 \mathrm{ppm})$. Aucune corrélation avec les autres éléments n’a été observée.

La concentration de l'Al oscille entre $3 \mu \mathrm{g}^{\bullet} \mathrm{L}^{-1}$ au puits $\mathrm{n}^{\circ} 1$ et $165 \mu \mathrm{g} \bullet \mathrm{L}^{-1}$ au puits $\mathrm{n}^{\circ} 13$ et demeure inférieure à la norme OMS. L'Al présente des corrélations positives avec le $\mathrm{Mn}, \mathrm{Ba}$, $\mathrm{Ca}$ et $\mathrm{Br}$, dont les valeurs de $\mathrm{R}^{2}$ sont successivement $(0,75,0,14$, $0,3$ et 0,02$)$. Des corrélations négatives sont constatées entre l'Al et le $\mathrm{Na}, \mathrm{K}, \mathrm{Zn}, \mathrm{Sr}$ et B. Ces corrélations montrent que la concentration de l'Al dans les eaux est le résultat de l'altération des couches carbonatées.

Les teneurs en Ba sont inférieures à la norme et varient entre 15 et $45 \mathrm{mg} \bullet \mathrm{L}^{-1}$. Les valeurs les plus élevées sont enregistrées dans les eaux des puits $\mathrm{n}^{\text {os }} 5,6$ et 7 . Il semble que cet élément dérive du même substrat que le $\mathrm{Ca}$, car il a un coefficient de corrélation égal à 0,62 . Le Ba présente également une corrélation positive avec le $K$ et négative avec le $\mathrm{Na}\left(R^{2}=0,09\right)$ (Figure 8). Il résulte des phénomènes d'échange cationique en particulier des argiles.

La présence du $\mathrm{Sr}$ dans les eaux de la nappe est notable; la concentration varie de 2,76 au puits $\mathrm{n}^{\circ} 8$ à $15,14 \mathrm{mg} \cdot \mathrm{L}^{-1}$ au puits $\mathrm{n}^{\circ} 19$. Le comportement géochimique du $\mathrm{Sr}$ est similaire à celui $\mathrm{du} \mathrm{Ca}$, en raison de leurs positions dans le tableau périodique et, notamment, leurs rayons ioniques très voisins (HEM, 1970). Cependant, le strontium est moins abondant que l'ion $\mathrm{Ca}^{2+}$ et peut occuper les sites libres dans le réseau de certains minéraux carbonatés, tels que la calcite, l'aragonite et la dolomite. Il peut également être incorporé dans le réseau cristallin du gypse et dans la structure des minéraux argileux
(EDMUNDS, 1981). La corrélation significative entre le $\mathrm{Sr}$ et les sulfates $\left(R^{2}=0,51\right)$ laisse penser que l'origine de Sr dans les eaux souterraines est due principalement à la dissolution du gypse. Il faut noter que les concentrations des différents éléments sont relativement élevées au niveau des puits $\mathrm{n}^{\text {os }} 6$ et 10. Ceci revient au fait que les murs de ces puits ont été construits avec des roches en provenance des terrils de la mine de fer assez riches en métaux.

La concentration de l'As est nettement inférieure à la norme OMS. La teneur minimale $\left(1,4 \mu \mathrm{g} \bullet \mathrm{L}^{-1}\right)$ est observée au puits $\mathrm{n}^{\circ} 5$, alors que le maximum 3,6 $\mu \mathrm{g}^{\bullet} \mathrm{L}^{-1}$ est enregistré au puits $\mathrm{n}^{\circ} 15$. Notons que dans notre cas d'étude (milieu oxydant $\left.=9 \mathrm{mg} \bullet \mathrm{L}^{-1}\right)$, l'As est présent à l'état d'oxydation +5 , il serait sous forme d'arsénite généralement complexé et adsorbé par les oxyhydroxides de fer. Ce processus est fonction du $\mathrm{pH}$ : plus le milieu est alcalin, plus l'adsorption des arsénites décroît (PLANT et al., 2003). L'As(V) est relativement immobile en raison de la coprécipitation avec $\mathrm{Fe}(\mathrm{OH})_{3}$ (BELZILE et TESSIER, 1990). Notons que l'adsorption par les hydroxydes de fer amorphes $\left[\mathrm{am}-\mathrm{Fe}(\mathrm{OH})_{3}\right]$ est un facteur contrôlant le comportement de l'As(V) et As(III) dans l'eau (SMEDLEY et KINNIBURGH, 2002). La corrélation négative observée entre le $\mathrm{Fe}, \mathrm{Mn}$ et As (Figure 8) montre qu'un apport en Fe et $\mathrm{Mn}$ réduit la concentration d'As dans les eaux (CASIOT et al., 2003; HALIM et al., 2009). L'As présente une corrélation positive avec le Ca et, à moindre degré, avec le K. Cependant, des corrélations significatives avec les éléments étudiés dans les terrils de la mine de Jérissa et dans le sol avoisinant sont observées. Ce résultat signifie que la présence de l'As, dans les eaux de la nappe, proviendrait de la dissolution des roches carbonatées et non des rejets miniers et des engrais.

La comparaison des concentrations des métaux dans les eaux de la nappe de K. Kh. avec celles d'autres systèmes aquifères montre que notre cas d'étude présente les concentrations les plus faibles malgré les énormes quantités de rejets et les teneurs élevées en métaux qui y sont contenus (Tableau 4). Ces résultats mettent en évidence le pouvoir auto-épurateur

Tableau 4. Teneur de quelques métaux $\left(\mu \mathrm{g}^{\circ} \mathrm{L}^{-1}\right)$ dans des nappes polluées. Table 4. Heavy metal levels $\left(\mu g^{\bullet} L^{-1}\right)$ in the polluted groundwater.

\begin{tabular}{|c|c|c|c|c|c|}
\hline Éléments & Maroc $^{1}$ & Espagne $^{2}$ & Jordani $^{3}$ & Tunisie $^{4}$ & Présente étude \\
\hline $\mathrm{Fe}$ & 444 & 80 & 527 & 227 & 128 \\
\hline $\mathrm{Mn}$ & 746 & 123 & 91 & 58 & 20 \\
\hline $\mathrm{Cu}$ & - & 90 & 110 & 21 & 4 \\
\hline $\mathrm{Cr}$ & 84 & 56 & 41 & 23 & 7 \\
\hline As & - & 2 & - & 10 & 3,6 \\
\hline $\mathrm{Pb}$ & 56 & 37 & - & 0,8 & 0,2 \\
\hline $\mathrm{Zn}$ & - & 8 & 36 & - & 112 \\
\hline
\end{tabular}

${ }^{1}$ CHOFQI et al., 2004; ${ }^{2}$ VILLALBA et al., 1995; ${ }^{3}$ AL-KHARABSHEH, 1999; ${ }^{4}$ BEN HAMZA, 1994. 
du sous-sol par la rétention des éléments métalliques. En effet, la couverture pédologique de la nappe phréatique de K. Kh. renferme des teneurs importantes en carbonate (20-40\%) et des quantités non négligeables de matière organique (5-12\%), ce qui entraîne la complexation et la rétention des éléments métalliques. Le même comportement a été souligné dans des études de cas soulevées par plusieurs auteurs (COSTA et al., 2002; COVELO et al., 2004; KOMAREK et al., 2009; KRAPAC et al., 2002; SIPOS et al., 2005). Contrairement à la pollution métallique, les polluants azotés par leurs charges négatives ont pu s'infiltrer à travers les couches non saturées et engendrer une élévation des taux des nitrates dans l'aquifère, comparativement à plusieurs études dans le monde (ALASMARI, 2006; MARKOVIC et al., 2006).

\section{CONCLUSION}

L'analyse de la qualité des eaux de la nappe de K. Kh. montre qu'elles sont globalement de bonne qualité physicochimique. Les faciès géochimiques les plus dominants sont $\mathrm{Ca}-\mathrm{Mg}-\mathrm{HCO}_{3}, \mathrm{Ca}-\mathrm{Mg}-\mathrm{SO}_{4}-\mathrm{HCO}_{3}$ à $\mathrm{Na}-\mathrm{Cl}$. Ces faciès sont fonction des affleurements géologiques et du temps de séjour des eaux dans les couches saturées.

L'analyse de la concentration des différents métaux dans les terrils et les sols environnants montre des teneurs élevées avec des corrélations significatives pour la majorité d'entre eux. Cependant, les teneurs de ces différents polluants dans les eaux de la nappe restent relativement faibles. Ce comportement semble être dû à l'adsorption de ces éléments par les différents constituants du sous-sol, notamment les carbonates et les matières organiques. La corrélation établie entre les différents éléments analysés montre que la totalité des métaux rencontrés dans les eaux provient principalement de la dissolution des couches géologiques. Toutefois, l'intensification de l'agriculture, la construction des ouvrages agricoles et la réalisation des pistes agricoles à partir des roches en provenance de la mine de Jérissa peuvent engendrer la contamination de la nappe. Dans ce contexte, il serait intéressant d'étudier avec précision, à l'aide d'essais de laboratoire contrôlés, les mécanismes intervenant dans le transport des métaux issus des terrils vers la nappe et le rôle joué par les différents constituants du sous-sol.

\section{BIBLIOGRAPHIE}

ACME ANALYTICAL LABORATORIES LTD. 852 E. Hastings ST. Vancouver V6A 1R6 Phone (604)253-3158 Fax (604)253-1716@ CSV Text Format.
AISSAOUI-ADJALI S., H. MAHJOUBI et S. TLIG (1988).

Origine épigénétique commune de la minéralisation en fer (sédirite) de deux types de gisements associés : stratiforme et karstique. Le cas du jbel Jérissa (Tunisie). C. R. Acad. Sci. Paris, Série II. 306 : 357-360.

ALASMARI M.N. (2006). Nitrate contamination of groundwater: A conceptual management framework. Environ. Impact Assess. Rev., 27, 220-242.

AL-KHARABSHEH A. (1999). Ground-water quality deterioration in arid areas: a case study of the Zerqa river basin as influence by Khirbet Es-Samra waste water (Jordan). J. Arid. Environ., 43, 227-239.

ANOUAR O., K. LASZLO, P. HANS-RUDOLF et C. FRED (2006). Rare earth elements abundance in Tunisian phosphorite. Dans : $4^{\text {th }}$ Swiss Geoscience Meeting, Bern, Switzerland, 2006, (affiche).

APPELO C.A.J. et D. POSTMA (1999). Geochemistry. Groundwater and Pollution. A.A. Balkema, Rotterdam. $535 \mathrm{p}$.

BEJI SASSI A. et S. SASSI (1999). Le cadmium associé aux dépôts phosphatés en Tunisie méridionale. J. Afr. Earth Sci., 29, 501-513.

BELZILE N. et A. TESSIER (1990). Interaction between arsenic and iron oxyhydroxides in lacustrine sediment. Geochim. Cosmochim. Acta 54, 103-109.

BENHAMZACH.(1994). Bilanhydrologique, sédimentologique et géochimique d'un versant en zone semi-aride. La Mejerda (Tunisie du Nord). Impact sur l'environnement. Doctorat en Sciences Géologiques. Université Pierre et Marie Curie. Paris VI, France, 283 p.

BOBOS I., N. DURÁES etF. NORONHA (2006). Mineralogy and geochemistry of mill tailings impoundments from Algares (Aljustrel), Portugal: implications for acid sulphate mine water formation. J. Geochem. Explor., 88, 1-5.

BOUHLASSA S. et A. AIACHI (2004). Origin of salinity of the shallow aquifer of Guelmime, South Morocco: Hydrogeochemical Study. Water Qual. Res. J. Canada, 39, 119-128.

BRIGATTI M.F., C. LUGLI et L. POPPI (2000). Kinetics of heavy metal removal and recovery in sepiolite. Appl. Clay Sci., 16, 45-57.

CAPPUYNS V., R. SWENNEN, A. VANDAMME et M. NICLAES (2006). Environmental impact of the 
former $\mathrm{Pb}-\mathrm{Zn}$ mining and smelting in East Belgium. J. Geochem. Explor., 88, 6-9.

CASIOT C., G. MORIN, F. JUILLOT, O. BRUNEEL, J.C. PERSONNÉ, M. LEBLANC, K. DUQUESNE, V. BONNEFOY et F. ELBAZ-POULICHET (2003). Bacterial immobilization and oxidation of arsenic in acid mine drainage (Carnoulès creek, France). Water Res., 37, 2929-2936.

CHOFQI A, A. YOUNSI, E.K. LHADI, J. MANIA, J. MUDRY et A. VERON (2004). Environmental impact of an urban landfill on a coastal aquifer (El Jadida, Morocco). J. Afr. Earth Sci., 39, 509-516.

COSTA J.L., H. MASSONE, D. MARTINEZ, E.E. SUERO, C.M. VIDAL et F. BEDMAR (2002). Nitrate contamination of a rural aquifer and accumulation in the unsaturated zone. Agric. Water Manage., 57, 33-47.

COVElO E.F., N. ÁLVAREZ, M.L. ANDRADE COUCE, F.A. VEGA et P. MARCET (2004). Zn adsorption by different fractions of Galician soils. J. Coll. I. Sci., 280, 343-349.

DGRE (Direction Générale des Ressources en Eau) (2005). Journée Mondiale de l'Eau. L'Eau et le Numérique, 22 mars 2005.

DUMESTRE A, S. SAUVÉ, M. MCBRIDE, P. BAVEYE et J. BERTHELIN (1999). Copper speciation and microbial activing in long-term contaminated soils. Arch. Environ. Contam. Toxicol., 36, 124-131.

EDMUNDS W.M. (1981). The hydrogeochemical characterisation of groundwaters in the Sirte basin, using strontium and other elements. Dans : The Geology of Lybia, SALEM, M.J. et M.T. BUSREWIL, (Éditeurs)., pp. 703-714. Academic Press, San Diego, CA, USA.

FETTER C.W. (1994). Applied Hydrogeology. Prentice Hall, Upper Saddle River, Bergen County, NJ, USA, 691 p.

FURRER G., B.L. PHILLIPS, K.U. ULTRICH, R. PÖTHIG et W.H. CASEY (2002). The origin of aluminium flocs in polluted streams. Science, 297, 2245-2247.

GNANDI K., G. TCHANGBEDJI, K. KILLI, G. BABA et K. ABBE (2006). The impact of phosphate mine tailings on the bioaccumulation of heavy metals in marine fish and crustaceous from the coastal zone of Togo. Mine Water Environ., 25, 56-62.
HADAS A, B. SAVING et N. HARUVY (1999). Agricultural practices, soil fertility management modes and resultant nitrogen leaching rates under semi-arid conditions. Agric. Water Manage., 42, 81-95.

HALIM M.A., R.K. MAJUMDER, S.A. NESSA, Y. HIROSHIRO, M.J. UDDIN, J. SHIMADA et K. JINNO (2009). Hydrogeochemistry and arsenic contamination of groundwater in the Ganges Delta Plain, Bangladesh. J. Hazard. Mater., 164, 1355-1345.

HATIRA N., V. PERTHUISOT et H. ROUVIER (1990). Les minéraux à $\mathrm{Cu}, \mathrm{Sb}, \mathrm{Ag} \mathrm{Hg}$ des minerais de $\mathrm{Pb}-\mathrm{Zn}$ de Sakiet Koucha (diapir de Sakiet Sidi Youssef, Tunisie septentrionale). Mineral. Deposita, 25, 112-117.

HEM D. (1970). Study and interpretation of the chemical characteristics of natural waters ( $2^{\text {nd }}$ edition). US Government Printing Office, Washington, DC, USA, 363 p.

HO J.C. (2001). Effect of land use and urbanization on hydrochemistry and contamination of groundwater from Taejon area. Korea. J. Hydrol., 253, 194-210.

JAUZIN A. (1967). Contribution à l'étude géologique de confins de la dorsale tunisienne (Tunisie septentrionale). Tunis : Secrétariat d'État au plan et à l'économie nationale, Division de la production industrielle.

KOMAREK M., A. VANEK, V. CHRASTNY, J. SZAKOVA, K. KUBOVA, P. DRAHOTA et J. BALIKA (2009). Retention of copper originating from different fungicides in contrasting soil types. J. Hazard. Mater., 166, 1395-14025.

KRAPAC I.G., W.S. DEY, W.R. ROY, C.A. SMYTH, S.L. SARGENT et J.D. STEELE (2002). Impacts of swine manure pits on groundwater quality. Environ. Pollut., 120, 475-492.

LANDREAU A. (1990). Pollution des eaux par les nitrates, Eau et Développement : Rev.Marocaine Eau, 10, 49-58.

LEE J.S. et H.T. CHON (2006). Hydrogeochemical characteristics of acid mine drainage in the vicinity of an abandoned mine. Daduk creek, Korea. J. Geochem. Explor., 88, 37-40.

MARKOVIC T., S. MIKO, S. KAPELJ, R. BULJAN, O. LARVA et Z. PEH (2006). Behaviour of metals and nutrients in soils and groundwater of a karst polje. J. Geochem. Explor., 88, 124-129. 
MATICIC B. (1999). The impact of agriculture on ground water quality in Slovinia: standards and strategy. Agric. Water Manage., 40, 235-247.

MCARTHUR J.M., D.M. BANERJEE, K.A. HUDSON-EDWARDS, R. MISHRA, R. PUROHIT, P.A. RAVENSCROFT, A. CRONIN, R.J. HOWARTH, C.A. HATTERJEE, T. TALUKDER, D. LOWRY, S. HOUGHTON et D.K. CHADHA (2004). Natural organic matter in sedimentary basins and its relation to arsenic in anoxic ground water: the example of West Bengal and its worldwide implications. Appl. Geochem., 19, 1255-1293.

MICHAUD A.M, M.N. BRAVIN, M. GALLEGUILLOS et P. HINSINGER (2007). Copper uptake and phytotoxicity as assessed in situ for durum wheat (Triticum turgidum durum L.) cultivated in Cu-contaminated former vineyard soils. Plant soil, 298, 99-111.

MLAYAH A., E. FERREIRA DA SILVA, F. ROCHA, CH. BEN HAMZA, A. CHAREF et F. NORONHA (2009). The Oued Mellègue: Mining activity, stream sediments and dispersion of base metals in natural environments, North-Western Tunisia. J. Geochem. Explor., $102,27-36$.

MLAYAH A., A. CHAREF, E. FERREIRA DA SILVA, F. NORONHA et CH. BEN HAMZA (2007). As Cu $\mathrm{Pb}$ and $\mathrm{Zn}$ contamination of stream sediments and their distribution in different size fractions (Jerissa mine - NW Tunísia). VI Congesso lberico de Geoquimica. Vila Real, Portugal. 16-21 juillet. $4 \mathrm{p}$.

PATNI N.K., L. MASSE, et P.Y. JUI (1998). Groundwater quality under conventional and no-tillage. Part I. Nitrate electrical conductivity and pH. J. Environ. Qual., 27, 869-876.

PETERSEN A.T., H.C.B. HANSEN et O. NYBROE (2004). Time and moisture effect on total and bioavailability copper in soil water extracts. J. Environ. Qual., 33, 505-512.

PLANT J.A., D.G. KINNIBURGH, P.L. SMEDLEY, F.M. FORDYCE et B.A. KLINCK (2003). Arsenic and selenium. Dans : Treatise on Geochemistry, H.D. HOLLAND et K.K. TUREKIAN (Éditeurs), 9, pp. 17-66.

PRATAS J., M.N.V. PRASAD, H. FREITAS et L. CONDEA (2005). Plants growing in abandoned mines of Portugal are useful for biogeochemical exploration of arsenic, antimony, tungsten and mine reclamation. J. Geochem. Explor., 85, 99-107.
RODIER J., C. BAZIN, J.P. BROUTIN, P. CHAMBON, H. CHAMPSAUR et L. RODI (1996). L'analyse de l'eau, eaux naturelles, eaux résiduaires, eau de mer. $8^{\mathrm{e}}$ édition. Dunod, Paris, France., 1383 p.

SALOMONS W. (1995). Environmental impact of metals derived from mining activities: processes, predictions, prevention. J. Geochem. Explor., 52, 5-23.

SIPOS P., T. NÉMETH, I. MOHAI et I. DODONY (2005). Effect of soil composition on adsorption of lead as reflected by a study on a natural forest soil profile. Geoderma., 124, 363-374.

SMEDLEY P.L. et D.G. KINNIBURGH (2002). A review of the source. Behaviour and distribution of arsenic in natural waters. Appl. Geochem., 17, 517-568.

STÄPFLI N. et C. MADRAMOOTOO (2006). Dissolved phosphorus losses in tile drainage under subirrigation. Water Qual. Res. J. Canada, 41, 63-71.

TLIG S., A. SASSI, H. BELAYOUNI et D. MICHEL (1987). Distribution de l'uranium, du thorium, du zirconium, $\mathrm{du}$ hafnium et des terres rares (Tr) dans des grains de phosphates sédimentaires. Chem. Geol., 62, 209-221.

TRABELSI R., M. ZAIRI et H. BEN DHIA (2007). Groundwater salinisation of the Sfax superficial aquifer. Hydrogeol. J., 15, 1341-1355.

USEPA (US Enviromental Protection Agency (1995). Drinking-water regulation and health advisories, Office of Water, Washington, DC, USA, 11 p.

VILLALBA M., F.J. BARRAGAN DE LA ROSA, M. TERNERO RODRÍGUEZ et J.C. JIMÉNEZ SANCHEZ (1995). Hydrochemical study of an aquifer system of an agricultural area in South-West Spain. Water. Res., 29, 1361-1372.

WEIL R.R., R.A. WEISMILLAR et R.S. TUNNER (1990). Nitrate contamination of groundwater under irrigated coastal plain soils. J. Environ. Qual., 19, 441-448.

WHO (2004). Guidelines for drinking water quality recommendations, Vol. 1, World Health Organization, Genève, Suisse, 515 p.

ZAIER A. (1999). Évolution tectono-sédimentaire du bassin phosphaté du Centre-Ouest de la Tunisie. Minéralogie, pétrographie, géochimie et genèse des phosphorites. Doctorat en Sciences Géologiques, Université de Tunis II, FST, Tunisie, $370 \mathrm{p}$. 
ZEBIDI H. (1976). Étude hydrogéologique de la nappe de

Kala-Khasba. Direction Générale des Ressources en Eau, Tunis, Tunisie, $37 \mathrm{p}$. 